\section{Die Kaufuntersuchung des Pferdes aus rechtlicher Sicht}

\author{
D. Plewa, Germersheim
}

\section{Einleitung}

Seit vielen Jahren befasst sich die Fachliteratur mit der Untersuchung von Pferden im Zusammenhang mit einem beabsichtigten oder vollzogenen Kauf (Eder 1999). Die tierärztliche Tätigkeit war speziell seit dem Grundsatzurteil des Bundesgerichtshofes (BGH) zur Veriährung von Schadensersatzansprüchen aus einer fehlerhaften Ankaufsuntersuchung (BGH 1983) Gegenstand zahlreicher Prozesse und gerichtlicher Entscheidungen. Statistisch nicht zu erfassen sind die nach den Erfahrungen des Autors sehr häufigen Fälle außergerichtlicher Regulierung von Schadensersatzansprüchen, die vom Käufer eines Pferdes gegenüber dem untersuchenden Tierarzt geltend gemacht werden. Die forensische Bedeutung der tierärztlichen Tätigkeit im Spannungsfeld zwischen den Interessen von Verkäufer und Käufer eines Pferdes wird durch die zum 01.01. 2002 wirksam gewordene Schuldrechtsreform, speziell die Neufassung des Kaufrechts möglicherweise noch zunehmen. Allerdings sind Befürchtungen, der Tierarz† habe nach Wegfall der Spezialvorschriften zum Viehkauf ( $\S \S$ 481 ff. BGB a.F.) den "schwarzen Peter" keineswegs begründet, ist doch der Käufer im Rahmen der ihm obliegenden Schadensminderungspflicht gehalten, zunächst einmal Ansprüche gegenüber dem Verkäufer geltend zu machen (OLG Schleswig 1987). Da durch das Kaufrecht never Fassung (BGB 2002) die Rechtstellung des Käufers deutlich gestärkt wurde, tritt jedenfalls dann die Rolle des Tierarztes in den Hintergrund, wenn nicht individual- oder formularvertraglich von der Gesetzeslage zu Gunsten des Verkäufers abgewichen wurde.

\section{Zur Terminologie}

In der Rechtsprechung findet sich sehr häufig der Begriff der Ankaufsuntersuchung, der sich im übrigen auch in der Praxis durchgesetzt hat (OLG Hamm 1996 et al.). Dabei soll es sich um die tierärztliche Untersuchung eines Pferdes handeln, deren Ergebnis - aufschiebende oder auflösende - Bedingung für das Zustandekommen eines Kaufvertrages wird (Schulze 1992, Fellmer 1990), wobei Auftraggeber regelmäBig der Käufer sein soll, ohne dass dies zwingend wäre (Schulze 1992, Eikmeier 1990). In der Literatur wurde vorgeschlagen, daneben die Begriffe Verkaufs- und Gewährschaftsuntersuchung zu verwenden. Dabei sollte unter Verkaufsuntersuchung eine unabhängig vom konkreten Rechtsgeschäft vor dem Kauf veranlasste tierärztliche Untersuchung, unter Gewährschaftsuntersuchung die tierärztliche Untersuchung nach dem Kauf im Hinblick auf eine vertraglich vereinbarte Gewährleistung zu verstehen sein (Eikmeier 1990, Schulze 1992).
Die Gewährschaftsuntersuchung

Die Gewährschaftsuntersuchung hat in der Praxis kaum eine Rolle gespielt. Wenn es nämlich um die Frage der gesetzlichen Haftung für Hauptmängel oder eine erweiterte vertragliche Haftung gem. § 492 BGB a.F. ging, wurde die tierärztliche Untersuchung regelmäßig zu Beweiszwecken oder aber - völlig unabhängig von der vertraglichen Haftungslage durch eine Erkrankung des gekauften Pferdes veranlasst. Die veterinärmedizinischen Feststellungen dienten dann zur Verfolgung von Ansprüchen gegenüber dem Verkäufer, und zwar als Grundlage für den Nachweis des Vorhandenseins eines Hauptmangels oder das Nichtvorhandensein einer zugesicherten Eigenschaft. Die Relevanz des Untersuchungsergebnisses betraf also das Rechtsverhältnis der Kaufvertragsparteien, nicht dasjenige zwischen Auftraggeber und Tierarzt.

\section{Die Verkaufsuntersuchung}

Der Begriff der Verkaufsuntersuchung findet sich zwar vereinzelt in Urteilen (OLG Oldenburg 1998), lässt aber eine inhaltliche Präzision insofern vermissen, als einerseits die Person des Auftraggebers als Unterscheidungsmerkmal nicht taugt, andererseits fraglich ist, welcher zeitliche Abstand zum Kaufvertragsabschluss gegeben sein muss, um nicht doch von einer "Ankaufsuntersuchung" ausgehen zu müssen. Es ist nämlich in der Rechtsprechung durchaus umstritten ist, ob nicht auch bei einer vor dem Kauf durchgeführten Untersuchung von einer Ankaufsuntersuchung im weiteren Sinne auszugehen ist mit der Konsequenz, dass sich durchaus die Frage der Haftung des untersuchenden Tierarztes bei einem unzutreffenden Untersuchungsergebnis stellt (OLG Köln 1992, OLG Düsseldorf 1998). Letztlich mag die Schöpfung verschiedener Begriffe jedenfalls für die Rechtsprechung eher verwirrend gewesen sein, weil die Aussagefähigkeit im Hinblick auf den Begriffsinhalt begrenzt war und die Bildung eines einheitlichen Verständnisses vom Begriff der Ankaufsuntersuchung eher erschwert wurde. Die Diskussion soll hier nicht vertieft, wohl aber soll klargestellt werden, dass das Unterscheidungskriterium zwischen Verkaufs- und Ankaufsuntersuchung schon nach bisheriger Terminologie (Fellmer 1990 et al.) nicht die Person des Auftraggebers war, konnte doch eine Ankaufsuntersuchung durchaus auch vom Verkäufer in Auftrag gegeben werden. Entscheidend war vielmehr der Bezug zu einem konkreten Rechtsgeschäft. Die Verkaufsuntersuchung diente eher der Vorbereitung eines beabsichtigten Verkaufs, die tierärztliche Bescheinigung über das Untersuchungsergebnis als Verkaufsargument, wobei die rechtliche Qualifizierung Probleme bereitete. Die Rechtsprechung wertete die Übergabe eines Attestes über eine Verkaufsuntersuchung teils als Zusicherung einer Eigenschaft im Sinne von $\S 492$ BGB a.F. (OLG Schleswig 1987 et al.), also als rechtsgeschäftliche Willenserklärung, teilweise als schlichte Wissenserklärung des Inhalts, dass das Pferd mit dem vom Tierarzt wiedergegebenen Ergebnis untersucht worden sei (LG Itzehoe 2000 et al.). Von einer Verkaufsuntersuchung sollte deswegen überhaupt nur dann die Rede sein, wenn der Verkäufer eines Pferdes im Hinblick auf den allgemein beabsichtigten Verkauf die tierärztliche Untersuchung des anzubietenden Pferdes in Auftrag gibt und das Ergebnis der Untersuchung als objektive Informationsgrundlage für einen beliebigen Kaufinteressenten dienen soll. Die rechtliche Konsequenz läge darin, dass der Tierarzt eine Haftung gegenüber dem 
Käufer nicht übernimmt, weil es an der Einbeziehung in den Schutzbereich des Vertrages fehlt (vgl. unten 5). Im Verhältnis zum Käufer würde die Erwähnung von Befunden im Untersuchungsprotokoll dessen Kenntnis im Sinne des § 442 BGB n.F. begründen, der Käufer könnte also an derartige, ihm durch die Überlassung des tierärztlichen Berichts mitgeteilte Befunde keine Ansprüche anknüpfen.

Innerhalb des Vertragsverhältnisses sollte der Hinweis des Verkäufers auf das Untersuchungsergebnis lediglich als objektive Information dahingehend verstanden werden, dass das Pferd mit dem aus dem Untersuchungsprotokoll ersichtlichen Ergebnis zu einem bestimmten Zeitpunkt untersucht wurde. Angesichts der strittigen rechtlichen Qualifizierung des tierärztlichen Berichts über eine Verkaufsuntersuchung sollte allerdings der Verkäufer ausdrücklich klar stellen, dass der Inhalt nicht zum Inhalt einer Beschaffenheitsvereinbarung im Sinne von § 434 I BGB gemacht wird. Gerade dadurch würde dann eine - aktuelle - Untersuchung nicht unbedingt entbehrlich werden.

\section{Die Kaufuntersuchung}

Im Gegensatz zur Verkaufsuntersuchung sollte von einer (An)Kaufsuntersuchung immer dann gesprochen werden, wenn sie in zeitlicher Nähe zu einem konkreten Verkaufsvorgang durchgeführt wird auf der Grundlage einer Vereinbarung zwischen Verkäufer und dem bereits individualisierten, also bekannten Käufer, dem das Untersuchungsergebnis als Teil der Grundlage seiner Kaufentscheidung dient. Dabei wird auch nach dem neuen Schuldrecht davon auszugehen sein, dass die Billigung des Pferdes als Kaufgegenstand in Kenntnis des Untersuchungsergebnisses aufschiebende oder auflösende Bedingung für das Zustandekommen des Kaufvertrages ist (Fellmer 1990 et al.). Dies bedeutet: Das Zustandekommen des - ansonsten bereits unbedingt - abgeschlossenen Vertrages hängt lediglich vom Untersuchungsergebnis ab oder aber der bereits abgeschlossene Vertrag wird durch das Untersuchungsergebnis rückwirkend unwirksam. Rechtliche Konsequenzen hat die unterschiedliche Konstellation bezüglich des Gefahrüberganges daneben möglicherweise auch für das Fortbestehen des Versicherungsschutzes aus einem Tierlebensversicherungsvertrag. Nach den allgemeinen Bedingungen für die Versicherung von Pferden dürfte der Versicherungsschutz bei einem auflösend bedingten Kauf enden, weil das Pferd aus dem Gewahrsam des Versicherungsnehmers "für davernd ausscheidet", was beim aufschiebend bedingten Kauf zweifelsfrei zu verneinen ist (AVP 1995). Empfehlenswert ist es sicherlich, das Zustandekommen des Kaufvertrages vom Untersuchungsergebnis abhängig zu machen, also eine aufschiebende Bedingung zu vereinbaren. Wird nichts abweichendes verabredet, ist der Käufer zur Abnahme des Pferdes und Bezahlung verpflichtet, wenn nach Treu und Glauben die Billigung des Pferdes erwartet werden kann (OLG Köln 1995 et al.).

\section{Ergebnis}

Mit dem Inkrafttreten des neven Kaufrechts sollte die Verwendung der Begriffe Gewährschafts- und Verkaufsuntersuchung entbehrlich geworden sein, jedenfalls wird ihre praktische Bedeutung weiter abnehmen. Das Gegenteil gilt aus nachstehend noch zu erörternden Gründen für die Ankaufs- oder
Kaufuntersuchung. Der Klarheit wegen sollte künftig von einer Kaufuntersuchung gesprochen werden. Die Verwendung des Begriffs Ankaufsuntersuchung hat allenfalls den Vorzug der Popularität, nicht dagegen den der größeren Aussagekraft, da schließlich zum An- auch immer der Verkauf gehört.

\section{Interessenlage nach altem und neuem Kaufrecht}

Die Kaufuntersuchung dient dem Pferdekäufer als wesentliche Entscheidungshilfe. Sie soll ihm, wenn auch im Rahmen einer Momentaufnahme (Huskamp 1987) einen Überblick über den Gesundheitszustand und mögliche Mängel verschaffen, die geeignet sind, die Leistungs- und Einsatzfähigkeit des Pferdes zu beeinträchtigen. Da nach $\S 482$ BGB a.F. der Verkäufer eines Pferdes grundsätzlich nur für Hauptmängel haftete, zudem der aufgeklärte Verkäufer eine erweiterte Gewährleistung für das Freisein von gesundheitlichen Beeinträchtigungen regelmäßig nicht übernahm, kam dem Ergebnis der Kaufuntersuchung eine entscheidende Bedeutung im Hinblick auf die Information des Käufers zu (Plewa 1987 et al.). Der Verkäufer hatte regelmäßig ein Interesse daran, dass möglichst keine Befunde entdeckt wurden, jedenfalls nicht solche, die den Käufer hätten abschrecken können. Der Käufer dagegen erwartete eine möglichst umfassende Aufklärung über den aktuellen Gesundheitszustand, regelmäßig verknüpft mit der Erwartung, im Sinne einer Prognose auch über künftige Auswirkungen vorhandener Befunde unterrichtet zu werden (OLG Hamm 1985).

Dem Verkäufer "alten Rechts" konnte es durchaus gelegen sein, wenn der Käufer erst gar nicht auf die Idee kam, eine Kaufuntersuchung zum Inhalt des Kaufvertrages zu machen. Insoweit hat sich die Interessenlage durch die Neufassung des Kaufrechts geändert: Der Verkäufer eines Pferdes haftet künftig für jede Abweichung von der vereinbarten Beschaffenheit, ansonsten für alle die nach dem Vertrag vorausgesetzte oder die gewöhnliche Verwendung beeinträchtigende Mängel, § 434 Abs. 1 BGB, soweit nicht die Haftung vertraglich ausgeschlossen ist. Den Möglichkeiten, von der gesetzlichen Vorgabe abzuweichen, sind für den Unternehmer im Sinne von § 14 BGB (neue Fassung) beim Verkauf an einen privaten Käufer, den Verbraucher im Sinne von § 13 BGB, enge Grenzen gesetzt. Bei anderen Vertragskonstellationen, also beim Verkauf von privat an privat oder von Unternehmer an Unternehmer, besteht dagegen im Rahmen der Vertragsfreiheit das Recht, einen vollständigen Gewährleistungsausschluss vorzusehen. In diesem Fall ebenso wie beim Verbrauchsgüterkauf gemäß § 475 BGB n.F. (Verkauf vom Unternehmer an den Verbraucher) gewinnt die Kaufuntersuchung an Bedeutung.

Der gewerbliche Verkäufer wird ein Interesse daran haben, dass im Rahmen einer Kaufuntersuchung festgestellte Befunde vom Käufer akzeptiert werden. Insoweit ist dann nämlich eine Haftung des Verkäufers wegen der Kenntnis des Käufers nach § 462 BGB ausgeschlossen.

Denkbar ist auch, dass als gesundheitliche Beschaffenheit des Pferdes der Zustand vereinbart wird, wie er sich nach dem tierärztlichen Untersuchungsprotokoll ergibt (FN 2002). Problematisch ist diese Vertragskonstellation allerdings, weil der Verkäufer durch die Beschaffenheitsvereinbarung auch für solche Mängel haften dürfte, die vom Tierarzt übersehen und deswegen im Untersuchungsprotokoll nicht aufgeführt wur- 
den, darüber hinaus aber auch für solche Mängel, auf die sich die Kaufuntersuchung gar nicht erstreckte. Die Bezugnahme auf die vom Tierarzt festgestellte gesundheitliche Beschaffenheit könnte nämlich dahingehend interpretiert werden, dass das Pferd andere als die in dem Untersuchungsbericht aufgeführten Befunde nicht aufweist.

Beim vertraglich vereinbarten Gewährleistungsausschluss hat der Käufer ein Interesse daran, umfassend über alle erheblichen Befunde aufgeklärt zu werden, um sich danach für oder gegen den Kauf entscheiden zu können. Da bei späterem Auftreten gesundheitlicher Beeinträchtigungen Ansprüche gegenüber dem Verkäufer ausscheiden, kommt als Anspruchsgegner ausschließlich der untersuchende Tierarzt in Betracht. Unverändert besteht natürlich das Interesse eines jeden Verkäufers, dass nicht solche Mängel entdeckt werden, die den Kaufinteressenten vom Erwerb abhalten. Andererseits ist ein gegenüber der früheren Gesetzeslage gesteigertes Interesse des Verkäufers an der Durchführung einer Kaufuntersuchung nicht zu leugnen, dessen Bedeutung sollte allerdings nicht überschätz† werden. Eine grundlegend veränderte Rolle hat die Kaufuntersuchung durch die Neufassung des Kaufrechts nicht erhalten. Die Brisanz für die tierärztliche Haftung wurde im Hinblick auf die erweiterten Käuferrechte sogar eher verringert.

\section{Das Vertragsverhältnis Tierarzt/Auftraggeber}

\section{Vertragstyp}

Es besteht kein Streit darüber, dass der Vertrag über die Durchführung einer Kaufuntersuchung als Werkvertrag zu qualifizieren ist (BGH 1983). Dies hat im Gegensatz zum tierärztlichen Behandlungsvertrag, der von Ausnahmen abgesehen als Dienstvertrag anzusehen ist (Köhler und Kraft 1984) zur Folge, dass nicht nur die Untersuchung an sich, sondern eine im Rahmen des erteilten Auftrages vollständige Untersuchung mit richtigem Ergebnis und schriftlicher Befundung geschuldet ist (Köhler und Kraft 1984). Die Autoren weisen in diesem Zusammenhang richtigerweise darauf hin, dass bei entsprechender Vereinbarung das Gutachten nur mündlich zu erstatten ist.

\section{Untersuchungsumfang}

Den Untersuchungsumfang bestimmt grundsätzlich der Auftraggeber (Köhler und Kraft 1984). Allerdings greift bereits hier die tierärztliche Aufklärungspflicht: Der Tierarzt isł gehalten, den Vertragspartner darüber zu unterrichten, in welchem Umfang üblicherweise Untersuchungen durchgeführt werden, wobei die Anforderungen an diese Informationspflicht umso höher sind, je unerfahrener der Auftraggeber ist und je praxisferner die von ihm gewünschten Einschränkungen des Untersuchungsumfanges sind. Zudem ist der Auftraggeber dann aufzuklären, wenn das Ergebnis eines bestimmten Untersuchungsschrittes die Durchführung einer weitergehenden Untersuchung zur Abklärung der Relevanz des erhobenen Befundes indiziert (Eikmeier 1990). Im Wesentlichen unproblematisch ist die rechtliche Beurteilung dann, wenn im Rahmen nachweisbarer Absprachen der Untersuchungsumfang individuell fest umrissen wurde. Klärunsbedürftig dagegen sind die Fälle, in denen lediglich eine Kaufuntersuchung an sich oder aber, um einen gängigen Jargon aufzugreifen, ein "kleiner" oder "großer TÜV" in Auftrag gegeben wurde. Letztere Unterscheidung ist insofern noch relativ klar, als mit dem "kleinen TÜV" die klinische, mit dem "großen" auch die röntgengologische Untersuchung gemeint ist. Es stellt sich allerdings die Frage, ob damit dann der Untersuchungsumfang hinreichend definiert ist. Mit anderen Worten: Gibt es einen standardisierten Untersuchungsumfang? Hiergegen wurde in einzelnen Gerichtsurteilen argumentiert, den könne es schon deswegen nicht geben, weil es einen klar definierten Begriff der Ankaufsuntersuchung nicht gäbe, vielmehr zwischen Verkaufs- und Ankaufs- und Gewährschaftsuntersuchung unterschieden werde (OLG Karlsruhe 1996). Dieses Argument überzeugt sicherlich in Anbetracht obiger Ausführungen zur Terminologie, die eine hinreichend klare Abgrenzung zulässt, nicht. Wohl aber lässt die Verwendung inhaltlich sehr unterschiedlich ausgestalteter Untersuchungsprotokolle in der tierärztlichen Praxis Zweifel daran aufkommen, ob von einem Standarduntersuchungsumfang ausgegangen werden darf. Erfreulicherweise hat sich das vom Hippiatrika-Verlag herausgegebene Formular (Lauk und Huskamp Hsgb.1987) am Markł seit vielen Jahren so weit durchgesetzt, dass von verschiedenen Sachverständigen, die in gerichtlichem Auftrag tätig waren, die Auffassung vertreten wurde, der darin wiedergebene Untersuchungsumfang sei als Mindest-Standard der klinischen bzw. röntgenologischen Kaufuntersuchung anzunehmen. In der Tat spricht die Verbreitung des Formulars und die Akzeptanz in der tierärztlichen Praxis dafür, dass das von hochrangigen Experten entwickelte Untersuchungsprotokoll unverändert einen tragfähigen Konsens in der Tierärzteschaft wiederspiegelt. Unabhängig von der Frage, ob rund 15 Jahre nach dem erstmaligen Erscheinen des Formulars in Details Ergänzungsbedarf bestehen mag, z.B. durch Ergänzung der klinischen Untersuchung um die Endoskopie der Atemwege und Erweiterung der Röntgenuntersuchung der Strahlbeine und Sprunggelenke um eine Ebene, ist es ein Gebot der Stunde, Einmütigkeit darüber zu wahren, dass es einen standardisierten Kaufuntersuchungsumfang gibt, oder aber einen solchen zu definieren.

\section{Haftungsrisiken}

Ansprüche wegen fehlerhafter Kaufuntersuchungen waren und sind sehr häufiger Gegenstand außergerichtlicher wie gerichtlicher Auseinandersetzungen zwischen Geschädigten und Tierarzł (Eder 1999). Der Anknüpfungspunkt für die Inanspruchnahme des Tierarztes liegt nach der gesetzlichen Terminologie in der Pflichtverletzung. Sie muss zu einem Schaden geführt haben, der im Rahmen des hier behandelten Werkvertrages regelmäßig durch einen nicht verhinderten Kauf herbeigeführt wird. Eher selten sind die Fälle, in denen der übervorsichtige Tierarzt durch Angabe eines nicht vorhandenen Befundes die Veräußerung des untersuchten Pferdes vereitelt. Die Detailbetrachtung der entschiedenen Fälle lässt als Anknüpfungspunkt für die tierärztliche Haftung eine Aufteilung in folgende Fallgruppen zu:

Zum vereinbarten oder als standardisiert zugrunde liegenden Untersuchungsumfang gehörende Untersuchungsschritte werden unterlassen, z. B. es wird keine Beugeprobe durchgeführt (OLG Frankfurt 1992).

Es werden Röntgenbilder mangelhafter Qualität angefertigt, 
die nicht ausreichend interpretierbar sind (OLG Zweibrücken 1999).

Es werden im Rahmen der klinischen Untersuchung wahrnehmbare Veränderungen, z. B. Operationsnarben oder auf grundsätzlich auswertbaren Röntgenbildern erkennbare Befunde nicht mitgteilt (OLG Hamm 1996).

Eine derartige Pflichtverletzung führt dann zur Schadensersatzpflicht, wenn der "vernünftige Käufer" bei Aufklärung über den - möglicherweise (Unterlassung) - vorhandenen Befund vom Kauf Abstand genommen hätte. So hat der Tierarzt bei einem fehlerhaft unterlassenen Untersuchungsschritt oder mangelnder Interpretierbarkeit der Röntgenaufnahmen nachzuweisen, dass bei durchgeführter Untersuchung oder Anfertigung aussagefähiger Röntgenbilder kein Befund erhoben worden wäre (OLG Hamm 1996). Da es sich um einen vertraglichen Schadensersatzanspruch handelt, besteht er zunächst im Verhältnis zwischen den Vertragspartnern. Unproblematisch ist dies, soweit der Käufer Auftraggeber des Tierarztes ist, da er bei fehlerhafter Untersuchung zugleich der Geschädigte ist. Im Ergebnis allerdings ändert sich auch dann nichts, wenn die Untersuchung vom Verkäufer in Auftrag gegeben wurde. Insoweit ist die Empfehlung an den Tierarzt, sich doch tunlichst vom Verkäufer beauftragen zu lassen, um einer Inanspruchnahme durch den Käufer zu entgehen, aus rechtlicher Sicht nicht hilfreich: Die Rechtsprechung hat den Käufer eines Pferdes stets in den Schutzbereich des zwischen Verkäufer und Tierarzł abgeschlossenen Werkvertrages einbezogen, wenn für den Tierarzł erkennbar war, dass das von ihm mitgeteilte Ergebnis Grundlage der Kaufentscheidung sein sollte (OLG Köln 1992). Der durch die Pflichtverletzung des Tierarztes Geschädigte hat einen Anspruch auf Ersatz des "Vertrauensschadens" (OLG Hamm 1996). Der Geschädigte, der einen für ihn nachteiligen Vertrag geschlossen hat, kann daher grundsätzlich verlangen, so gestellt zu werden, wie er ohne das schuldhafte Verhalten seines Vertragsgegners - also ohne Zustandekommen des Vertrages stehen würde. Dies bedeutet: Er hat einen Anspruch auf Befreiung von dem abgeschlossenen Vertrag und auf Ersatz seiner nutzlos gewordenen Aufwendungen (OLG Hamm 1996). Der Tierarzt hat also dem Käufer den Kaufpreis zu ersetzen und alle im nachhinein nutzlos gewordenen Aufwendungen für Unterstellung, Fütterung, Pflege, tierärztliche Untersuchung und Behandlung sowie Hufbeschlag des Pferdes, kann aber zu seinem zweifelhaften Trost darauf bestehen, dass an ihn das Pferd herausgegeben wird. Dieser sehr umfangreichen Schadensersatzpflicht und dem geschilderten Haftungsrisiko sah sich der Tierarzt nach alter Rechtslage gemäß der Grundsatzentscheidung des BGH (BGH 1983) über eine 30 Jahre davernde Veriährungsfrist ausgesetzt. Seit dem 01.01.2002 gilt insoweit die allgemeine Veriährungsfrist von drei Jahren ( $\S \S 634 a \mid$ Z.3, 195 BGB ff.), soweit nicht vertraglich etwas anderes vereinbart ist (vgl. nachfolgend unter Z.6). Die Veriährung beginnt erst mit dem Schluss des Jahres, in welchem der Anspruch entstanden ist und der Auftraggeber "von den den Anspruch gegründenden Umständen" Kenntnis erlangt hat (§ 199 Abs. 1 Z.1,2 BGB).

\section{Haftungsauschluss und Haftungsbeschränkungen}

Das aus der geschilderten Rechts- und Gesetzeslage sich ergebende Haftungsrisiko des Tierarztes zwingt zu der Über- legung, ob und bejahendenfalls in welchem Umfang eine Einschränkung oder gar ein Ausschluss vertraglich vereinbart werden kann. Bei der Beantwortung dieser Frage ist im Wesentlichen zwischen individualvertraglichen und formularvertraglichen Vereinbarungen zu unterscheiden.

\section{Individualvertragliche Vereinbarungen}

Für Haftungsbeschränkungen, die individuell zwischen den Vertragsparteien ausgehandelt werden, gilt zwar das Prinzip der Vertragsfreiheit, jedoch mit erheblichen Einschränkungen. Zum Einen sind selbstverständlich gesetzliche Verbote einzuhalten, etwa die Bestimmung des $\S 202$ BGB neue Fassung, wonach die Verjährung für Haftung wegen Vorsatzes nicht verkürzt werden darf. Ebenso einzuhalten ist aber auch das Standesrecht, zumal es im Rahmen einer rechtlichen Überprüfung individualvertraglicher Absprachen als Maßstab für die Anwendung des Grundsatzes von Treu und Glauben ( $\S$ 242 BGB) Anwendung findet. In der Rechtsprechung ist anerkannt, dass individuelle Vertragsvereinbarungen auf ihre Angemessenheit hin kontrolliert werden können. Wird die Vertragsfreiheit von einer Partei zur Durchsetzung einseitiger Interessen genutzt, können unangemessene Vertragsregelungen im Einzelfall unwirksam sein. Vor diesem Hintergrund muss mit Sicherheit davon ausgegangen werden, dass etwa die Haftung des Tierarztes für die Verletzung elementarer Pflichten oder grobes Verschulden ebensowenig eingeschränkt werden kann wie insoweit eine Verkürzung der Verjährung auf weniger als ein Jahr in Betracht kommt. Darüber hinaus ist zu beachten, dass die Gerichte speziell bei Abweichungen von der gesetzlichen Veriährungsregelung einen strengen Maßstab anlegen und es sicherlich nicht hinnehmen werden, dass eine Umgehung der für Formularverträge geltenden Bestimmungen durch Schein-Individualvereinbarungen erreicht werden soll (Mansel und Budzikiewicz 2002).

\section{Haftungsvereinbarungen in Formularverträgen}

Ohnehin spielen im Vertragsverhältnis zwischen Tierarzł und Auftraggeber individuelle Absprachen selten eine Rolle. Gerade bei der Kaufuntersuchung liegt die praktische Relevanz in der Anwendung eines Formularvertrages, wie er bereits in der Vergangenheit gängig war (Lauk und Huskamp 1987). In dem bereits erwähnten Formularvertrag war eine Verkürzung der Veriährungsfrist auf sechs Monate vorgesehen. Diese Regelung ist von der Rechtsprechung ausdrücklic gebilligt worden (OLG Oldenburg 1998). Nach der neuen Gesetzeslage ist eine Verkürzung der Veriährungsfrist im Rahmen von Formularverträgen lediglich noch auf ein Jahr zulässig, §§ 634a, 307 BGB (Henssler 2001). Erwähnenswert ist in dem Zusammenhang, dass die Verjährungsfrist nicht bereits mit dem Tage der Ankaufsuntersuchung beginnt, sondern entsprechend $\S 199$ BGB erst mit dem Ende des Jahres, in welchem der Anspruch entstanden ist und der Auftraggeber von dem Wesentlichen, den Anspruch begründenden Umständen Kenntnis erlangt hat.

Sind schon die Möglichkeiten begrenzt, die Verjährungsfrist zu verkürzen, so gewinnt die Überlegung an Relevanz, die Schadensersatzpflicht der Höhe nach einzuschränken. Hierzu besteht umso mehr Veranlassung, als immer noch die Rela- 
tion zwischen dem durchschnittlichen tierärztlichen Honorar für die Durchführung einer Kaufuntersuchung zu dem übernommenen Haftungsrisiko in einem nicht annähernd ausgeglichenen Verhältnis steht, welches mit steigendem Kaufpreis zunehmend aus den Fugen gerät. Vom Grundsatz her ist eine Beschränkung der Haftungssumme in einem Formularvertrag, etwa auf einen Betrag von 50.000 EUR, durchaus zulässig. Es gelten insoweit jedoch erhebliche Einschränkungen:

Ein Haftungsausschluss ist unwirksam, wenn er sich auch auf die Fälle von Vorsatz und grobe Fahrlässigkeit bezieht. Für den Tierarzł gilt darüber hinaus, dass er sich nicht für die Verletzung sogenannter Kardinalpflichten freizeichnen kann (Plewa 1987). Dies bedeutet, dass eine Begrenzung der Haftungssumme nur für leicht fahrlässige Verletzungen unwesentlicher Vertragspflichten wirksam ist (OLG Stuttgart 1992). Der praktische Anwendungsbereich erweist sich danach sicherlich als recht klein, andererseits sind durchaus Fälle denkbar, in denen die Haftungssummenbegrenzung greift, z.B. das Nichterwähnen eines Befundes der Gruppe II des Protokolls der Röntgenkommission, wenn dieser Befund innerhalb der Verjährungsfrist nicht klinisch relevant geworden ist. Wurden wirksam zwischen Verkäufer und Tierarzł Haftungsbeschränkungen vereinbart, so gelten diese auch im Verhältnis zum Käufer, soweit dem grundsätzlich Ansprüche aus dem Gesichtspunkt der Schutzwirkung des Vertrages zustehen (OLG München 1994).

\section{Fazit}

Es liegt im Interesse der gesamten Tierärzteschaft, die Anforderungen an eine "übliche" Kaufuntersuchung zu standardisieren. Diesem Ziel wird in der Praxis durch die Verwendung möglichst einheitlicher Untersuchungsprotokolle zu entsprechen sein, deren Inhalt dem aktuellen Stand der veterinärmedizinischen Wissenschaft und allgemein anerkannten Anforderungen an den Umfang der klinischen wie röntgenologischen Untersuchung zu entsprechen hat. Die Möglichkeiten des Tierarztes, durch einen Formularvertrag seine Haftungsrisiken einzuschränken, sind durch gesetzliche Vorgaben sehr begrenzt. Diesem Umstand sollte durch Abschluss einer Vermögensschadenhaftpflichtversicherung mit ausreichender Deckungssumme entsprochen werden, im Einzelfall kann eine individualvertragliche Vereinbarung hilfreich sein.

Im Spannungsfeld der Interessen zwischen Verkäufer und Käufer eines Pferdes kommt dem Tierarzł eine sehr wichtige Rolle zu. Die seit dem 01.01.2002 geänderte Gesetzeslage hat zur Folge, dass der Tierarzt vermehrt auch die Interessen des Verkäufers zu beachten hat. Potentielle Anspruchsteller sind Verkäufer wie Käufer unabhängig davon, wer Auftraggeber ist. Die haftungsrechtliche Relevanz der Kaufuntersuchung ist indes durch die Reform des Kaufrechts nicht erhöht, sondern im Hinblick auf die erhebliche Stärkung der Käuferrechte eher verringert worden.

\section{Literatur}

AVP (1995): Allgemeine Bedingungen für die Versicherung von Pferden

BGB (Bürgerliches Gesetzbuch) alte Fassung $\S \S 481$ ff. in Verbindung mit $\S 1$ ViehmängelVO
BGB (2002): Bürgerliches Gesetzbuch vom 02.01. 2002 BGBI. I, 122

BGH (1983): NJW 83, 2078

BGH (1986): NJW-RR 86, 1438

Deutsche Reiterliche Vereinigung (2002): FN-Formularvertrag, FNVerlag

Eder H. (1999): Pferdehaltung im deutschen Recht. Dissertation München. 111

Fellmer E., H. Eikmeier und H. Mögle (1990): Lehrbuch der gerichtlichen Tierheilkunde. Berlin und Hamburg, 65

Henssler M. und F. Graf von Westphalen (2001): Praxis der Schuldrechtsreform. ZAP Verlag, Recklinghausen

Huskamp, B. (1963): Der praktische Tierarzt. $141 \mathrm{f}$

Lauk H.D. und B. Huskamp Hsgb.(1987): Vertrag über die Untersuchung eines Pferdes, Hippiatrika Verlag, Stuttgart

Köhler H. und H. Kraft (1984): Gerichtliche Veterinärmedizin. Ferdinand Enke Verlag, Stuttgart

LG Fulda (1998): $2 \bigcirc 486 / 98$

LG Heidelberg (1994): $8 \bigcirc 294 / 94$

LG Itzehoe (2000): $2 \bigcirc$ 438/2000

LG Karlsruhe (1996): $8 \bigcirc$ 126/96

LG Lüneburg (1998): 1 S 24/98

LG Memmingen (1994): $3 \bigcirc 955 / 94$

LG Münster (1992): $10 \bigcirc 211 / 92$

LG Saarbrücken (1996): 11 S 299/96 bzgl. sechs Monate altem Attest

Mansel H. und C. Budzikiewicz (2002): Das neue Verjährungsrecht. Deutscher Anwaltverlag, Bonn, $190 \mathrm{f}$

OLG Düsseldorf (1998): $8 \cup 151 / 98$

OLG Frankfurt (1992): 23 U 44/92

OLG Hamm (1980): 24 U 164/80

OLG Hamm (1983): 24 U 215/83

OLG Hamm (1996): NJW-RR 96, 736, 737

OLG Karlsruhe (1996): 1 U 228/96

OLG Karlsruhe (1997): $6 \cup 38 / 97$

OLG Köln (1987): 13 U 16/87

OLG Köln (1992): VersR 92, 978

OLG Köln (1995): "NJW-RR 95", 113

OLG München (1994): 25 U 4042/94, VersR 96, 731

OLG Oldenburg (1998): VersR 98, 1563

OLG Schleswig (1987): VersR 87, 624

OLG Stuttgart (1992): VersR 92, 979

OLG Zweibrücken (1999): 5 U 9/99

Palandt (2002): Bürgerliches Gesetzbuch. Verlag Beck, 61. Auflage 2002, Rz 8 vor $\S 631$ BGB

Plewa D. (1987): „Vertrag über die Untersuchung eines Pferdes" - Ein Beitrag zur Standardisierung der Ankaufsuntersuchung beim Pferd. Teil I: Juristische Anmerkungen. Pferdeheilkunde 3, 297 - 302

Plewa D. (2001): Die Haftung des Tierarztes für Vermögensschäden im Zusammenhang mit der Ankaufsuntersuchung. Pferdespiegel $17 f f$.

Schulze E. (1992): Die Haftung des Tierarztes. Selbstverlag Berlin, $121 \mathrm{f}$.

von Salis B. (1996): Ankaufsuntersuchung beim Pferd. Gustav Fischer Verlag Jena

Dr. jur. Dietrich Plewa

Ludwig Erhard Straße 4

76726 Germersheim

E-Mail: rae.plewa-doppler@t-online.de 


\section{Leitsätze aus der Rechtsprechung zur Kaufuntersuchung eines Pferdes}

\author{
D. Plewa, Germersheim
}

In verstärktem Maße hat sich die Rechtsprechung seit dem Urteil des Bundesgerichtshofes aus dem Jahre 1983 (BGH 1983) in zahlreichen Entscheidungen mit Problemen rund um die tierärztliche Untersuchung eines Pferdes im Hinblick auf einen vollzogenen Verkauf befasst. Mit diesem Beitrag soll der Vesuch unternommen werden, einige der wichtigsten Urteile in Kurzfassung wiederzugeben, entweder durch Zitat der amtlichen Leitsätze oder Wiedergabe der wesentlich erscheinenden Feststellungen aus den Urteilsgründen. Ausgesucht wurden solche Urteile, die auch nach Inkrafttreten des neuen Schuldrechts (BGB 2002) Bedeutung haben.

\section{Vertragstyp und Untersuchungsumfang}

Der Vertrag, der die Durchführung einer tierärztlichen Kaufuntersuchung zum Gegenstand hat, ist Werkvertrag (OLG Hamm 1996).

Der Auftraggeber hat den Umfang der Kaufuntersuchung darzulegen und nachzuweisen, insbesondere den Beweis zu führen, dass neben einer klinischen auch eine röntgenologische Untersuchung gefordert war (LG Mönchen-Gladbach 1996).

Selbst dann, wenn der Tierarzt im Rahmen einer Kaufuntersuchung Röntgenaufnahmen gefertigt hat, obliegt es dem Auftraggeber, den Nachweis zu führen, dass auch Oxspring-Aufnahmen anzufertigen waren. Insoweit greifen weder Beweiserleichterungen noch eine Umkehr der Beweislast ein (OLG Karlsruhe 1996, Anm.: Die Rechtsauffassung erscheint jedenfalls dann sehr problematisch, wenn man von einem standardisierten Umfang der - röntgenologischen - Kaufuntersuchung ausgeht).

Die Anfertigung und Befundung von Röntgenbildern gehört nicht zum üblichen Umfang einer Kaufuntersuchung, zumal wenn sich bei der allgemeinen Untersuchung des Bewegungsappartes kein Hinweis auf eine Erkrankung ergibt. Es bedarf einer besonderen Beauftragung, die vom Auftraggeber nachzuweisen ist (OLG Karlsruhe 1997).

Die Anfertigung von Röntgenaufnahmen gehört nicht zum „"normalen Programm" einer Kaufuntersuchung (OLG Saarbrücken 1994).
Vertragspflichten

Der Tierarzt hat den Auftraggeber über den gesundheitlichen Zustand des Reitpferdes richtig und umfassend zu informieren (OLG Hamm 1983).

Der untersuchende Tierarzt hat die Aufgabe, Krankheitsbefunde und sonstige Unregelmäßigkeiten nach den Regeln der tierärztlichen Kunst festzustellen und dem Auftraggeber mitzuteilen; ein Zu- oder Abraten hinsichtlich des Kaufentschlusses gehört - zumindest ungefragt - nicht zu seinen Aufgaben (OLG Karlsruhe 1994).

Geschuldet ist vom Tierarzt die Aufklärung über nicht ganz fernliegende Risiken aus Befunden, die im Rahmen der Kaufuntersuchung erhoben werden, auch wenn die diagnostizierte Veränderung am Tag der Untersuchung noch keinen Krankheitswert hat (OLG Köln 1987).

Obwohl nur eine Soll-Empfehlung (Anm.: gemäß dem Protokoll der Röntgenkommission vom 14.04./01.07.1993) hat der Tierarzt die Pflicht, seinen Vertragspartner über eine Röntgenveränderung gemäß der Gruppe II aufzuklären, jedenfalls dann, wenn für den Tierarzt erkennbar ist, dass es sich um einen Umstand handelt, der für die Willensbildung des Vertragspartners von ausschlaggebender Bedeutung ist und den Vertragszweck vereiteln oder erheblich gefährden kann (LG Flensburg 2000).

Der Tierarzt hat einerseits den erhobenen Befund mitzuteilen und bei Röntgenveränderunngen darauf hinzuweisen, dass er eine Prognose nicht abgeben kann und die zukünftige Entwicklung der Eignung des Pferdes im Hinblick auf einen vorhandenen Befund (Anm.: hier: Veränderungen im Sinne von Prodotochlose) völlig unsicher ist.

Der Tierarzt ist verpflichtet, aussagekräftige, interpretierbare Röntgenbilder zu fertigen oder aber seinen Auftraggeber auf die beschränkte Aussagekraft aufmerksam zu machen. Verletzt er die Verpflichtung, hat er den Nachweis zu führen, dass zum Zeitpunkt der Untersuchung infolge der mangelnden Qualität der Röntgenaufnahmen nicht erkennbare, aber später festgestellte Röntgenveränderungen nicht vorhanden waren (OLG Zweibrücken 1999).

Im Rahmen einer klinischen Kaufuntersuchung darf auf die Durchführung der Zehengelenksbeugeprobe nicht verzichtet werden, sie ist unabhängig vom konkreten Auftragsumfang unverzichtbarer Bestandteil (OLG Frankfurt 1992).

\section{Kausalität}

Die Pflichtverletzung des Tierarztes ist für den Kaufentschluss ursächlich, wenn ein "vernünftiger" Käufer bei ordnungsgemäßer Ankaufsuntersuchung vom Kauf des Pferdes Abstand genommen hätte (OLG Hamm 1996).

Ist der Tierarzt lediglich beauftragt, ein Pferd klinisch zu unter- 
suchen und Röntgenbilder anzufertigen, so ist die Anfertigung einer für eine Befundung nicht ausreichenden Röntgenaufnahme für den kaufentschluss nicht ursächlich, wenn die endgültige Befundung einem anderen Tierarzt vorbehalten ist (OLG Karslruhe 1997).

\section{Umfang der Ersatzpflicht}

Zu ersetzen ist der Vertravensschaden. Der Geschädigte ist so zu stellen, wie er ohne das Zustandekommen des für ihn nachteiligen Vertrages stehen würde. Es sind somit der Kaufpreis und die nutzlos gewordenen Aufwendungen zu ersetzen (OLG Hamm 1996).

\section{Vertrag mit Schutzwirkung für Dritte}

Stellt ein Tierarzł im Auftrag des Verkäufers eines Reitpferdes ein Verkaufsattest aus mit der dem Tierarzt bekannten Bestimmung, auch dem Käufer als Grundlage für die Kaufentscheidung Aufschluss über die gesundheitliche Verfassung des Pferdes zu geben, so ist der Käufer in den Schutzbereich des Vertrages zwischen Tierarzł und Verkäufer einbezogen (OLG Köln 1992).

Voraussetzung für die Einbeziehung des Dritten in den Schutzbereich ist jedoch, dass sich Anhaltspunkte für einen auf den Schutz des Dritten gerichteten Parteiwillens ergeben. Dies ist in der Regel zu bejahen, wenn das Gutachten erkennbar für einen Dritten bestimmt ist (OLG Düsseldorf 1998).

Die Bejahung einer Schutzpflicht setzt allerdings nicht voraus, dass der Tierarzł die Zahl oder den Namen der zu schützenden Personen kennt. Es genügt vielmehr, dass ihm bekannt war, dass sein Gutachten/Untersuchungsergebnis für einen (potentiellen) Käufer bestimmt ist (LG Memmingen 1997).

Der Tierarzł muss, damit die Einbeziehung Dritter in den Vertrag nicht uferlos ausgedehnt wird, erkennen können, dass das Gutachten für einen - wenn auch irgendeinen - Käufer diente, die Personengruppe des "Dritten" muss klar abgrenzbar sein (LG Dortmund 1989).

Auch bei einem Vertrag mit Schutzwirkung zugunsten Dritter kann sich der Schuldner gegenüber einem Anspruch des Dritten auf eine diesem unbekannte, unter den Vertragsparteien wirksam vereinbarte Veriährungsfrist (Anm.: entsprechendes gilt für eine Haftungsbeschränkung) berufen (OLG Oldenburg 1998).
Auf die Schutzwirkung des Untersuchungsvertrages für den Käufer als Nicht-Auftraggeber kommt es nicht an, wenn dem Tierarzt ein sittenwidriges Handeln im Sinne des $\S 826$ BGB vorzuwerfen ist. Als sittenwidrige Schädigung ist zu werten, wenn ein Tierarzt ohne Entnahme einer Gewebsprobe eine walnussgroße Verdickung im Bereich des Unterkiefers eines wertvollen Pferdes als gutartig attestiert und insofern grob leichtfertig handelt (BGH 1985).

\section{Verkürzung der Verjährung/Haftungsvereinbarungen}

Nach der bis zum 31.12.2001 geltenden Gesetzeslage (§ 9 AGBG) war die formularmäßige Verkürzung der Veriährungsfrist auf sechs Monate zulässig. Eine solche Verkürzung gilt auch im Verhältnis zum in den Schutzbereich des Vertrages einzubeziehenden Dritten (OLG München 1994).

\section{Literatur}

BGH (1983): NJW 83, 2078

$B G H$ (1985): X ZR 95/85

BGB (2002): Bürgerliches Gesetzbuch, 122

LG Dortmund (1989): $3 \bigcirc 218 / 89$

LG Flensburg (2000): $3 \bigcirc$ 148/00

LG Hannover (1992): $18 \bigcirc 52 / 92$

LG Heidelberg (1994): $8 \bigcirc$ 294/94

LG Memmingen (1997): 3 ○ 955/97

LG Mönchen-Gladbach (1996): $3 \bigcirc 534 / 96$

LG Münster (1992): $10 \bigcirc 211 / 92$

OLG Düsseldorf (1998): 8 U 151/98

OLG Frankfurt (1992): 23 U 44/92

OLG Hamm (1996): NJW-RR 96, 736

OLG Hamm (1983): 24 U 215/83

OLG Karlsruhe (1994): 18a U 42/94

OLG Karlsruhe (1997): 6 U 38/97

OLG Karlsruhe (1996): 1 U 228/96

OLG Köln (1987): 13 U 16/87

OLG Köln (1992): VersR 92, 978

OLG München (1994): 25 U 4042/94

OLG Oldenburg (1998): VersR 98, 1563

OLG Saarbrücken (1994): 7 U 149/94

OLG Zweibrücken (1999): 5 U 9/99

Dr. jur. Dietrich Plewa

Ludwig Erhard Straße 4

76726 Germersheim

E-Mail: rae.plewa-doppler@t-online.de 


\section{Die rechtlichen Aspekte der Kaufuntersuchung in der Schweiz}

\author{
B. Krenger und R. Straub \\ Klinik für Nutztiere und Pferde, Unversität Bern
}

\section{Das rechtliche Verhältnis des Tierarztes zu seinen Kunden}

Das Verhältnis zwischen dem Tierarzt, der eine Kaufuntersuchung durchführen soll, und seinem Kunden ist ein rechtlich fassbarer Vertrag, unabhängig davon, ob ein schriftliches Vertragsdokument erstellt wird oder, was wohl meistens zutrifft, der Vertragsabschluss mündlich erfolgt.

Die Gerichtspraxis unterstellt den Vertrag zwischen dem Tierarzt und seinem Kunden dem Auftragsrecht, geregelt in den Artikeln 394 bis 406 des Obligationenrechtes. Durch die Annahme eines Auftrages verpflichtet sich der Beauftragte, die ihm übertragenen Geschäfte oder Dienste vertragsgemäß zu besorgen; er haftet dem Auftraggeber für getreve und sorgfältige Ausführung. Der Beauftragte erfüllt den Vertrag, wenn er sorgfältig und gewissenhaft, im Falle des Tierarztes auch nach dem neuesten Wissensstand, arbeitet. Diese rechtliche Qualifikation gilt sicher für den Behandlungsvertrag; ob für die Ankaufsuntersuchung allenfalls Werkvertragsrecht zur Anwendung kommen könnte, ist nach der Praxis offen. So oder so lassen sich aber aus rechtlicher Sicht die folgenden Ratschläge zur Erfüllung des Vertrages geben.

\section{Konsequenzen in der Praxis}

Grosse Sorgfalt ist dem Abschluss des Vertrages zu widmen. Insbesondere muss dessen Inhalt möglichst exakt festgelegt werden. Die Annahme eines allgemeinen Auftrages, an dem bestimmten Pferd eine Kaufuntersuchung durchzuführen, genügt nicht. Der Tierarzt hat als Fachmann die Pflicht, den Kunden über die Möglichkeiten verschiedener Untersuchungsmethoden aufzuklären. Aufgrund der sauberen Aufklärung ist dann der Umfang der Untersuchung festzulegen, es ist exakt zu vereinbaren, ob beispielsweise nur eine klinische Untersuchung gemacht werden soll oder ob diese auch bildgebende Diagnostik, wenn ja welche, umfassen soll. Ausdrücklich ist der Kunde auf Gefahren, die mit bestimmten Untersuchungsmethoden verbunden sind, hinzuweisen, so beispielsweise auf die Gefahren einer Narkose.

Wir halten es für sinnvoll, den Auftrag zur Kaufuntersuchung schriftlich festzuhalten, am ehesten mit einem Formular. Abgesehen vom medizinischen Umfang sollte das Formular auch den Preis der vereinbarten Untersuchung enthalten. Denkbar wäre, auf diesem Formular in geeigneter Form eine Beschränkung der Haftung abzumachen.

In der Ausführung des Auftrages umfasst die Sorgfaltspflicht nicht nur die eigentliche veterinärmedizinische Untersuchung, deren Qualität nicht durch die Juristen, sondern durch die Tierärzte zu definieren ist, sondern auch die Formulierung des schriftlichen Berichtes. Dieser Bericht muss vollständig sein und klar zum Ausdruck bringen, was untersucht wurde, damit auch feststeht, was nicht geprüft wurde. Unsicherheiten sind offen zu legen: Wird ein Pferd nur klinisch untersucht, muss, wo angezeigt, im Bericht darauf hingewiesen werden, dass das Resultat nur beschränkt Gültigkeit hat; so denken wir, dass zum Beispiel bei Anzeichen von Spat die Empfehlung nötig ist, dass die Untersuchung durch Röntgenaufnahmen ergänzt werden sollte.

Führt man mit der Ankaufsuntersuchung keinen "Dopingtest" durch, kann man sich in der heutigen Zeit fragen, ob der Bericht nicht auch einen Hinweis darauf enthalten sollte, dass nicht überprüft wurde, ob das Pferd zur Zeit der Untersuchung unter dem Einfluss von Medikamenten stand.

Deutliche Erwähnung sollte die Tatsache finden, dass eine Ankaufsuntersuchung immer nur eine Momentaufnahme ist und eine Prognose über die Entwicklung des Pferdes in gesundheitlicher Sicht nicht gegeben werden kann. Diese Feststellung könnte auch in eine Klausel zur Beschränkung der Haftung eingeschlossen werden.

\section{Der Vertragspartner des Tierarztes}

Bei einer Kaufuntersuchung wird normalerweise, wie dies der Begriff ja andeutet, der Käufer eines Pferdes Auftraggeber und damit Vertragspartner des untersuchenden Tierarztes sein. Diese Konstellation ist für beide Vertragspartner kaum problematisch, sind doch die Interessen gleich gerichtet. Der Käufer des Pferdes ist an einer möglichst sorgfältigen Untersuchung mit klarer Darstellung des gesundheitlichen Zustandes des zu kaufenden Pferdes, allfälliger gesundheitlicher Mängel und der Risiken interessiert, ein Interesse, das dem Tierarzł uneingeschränkte Sorgfalt zulässt.

Vorsicht ist für den Tierarzł geboten, wenn sich abzeichnet, dass der Käufer, meist aus emotionalen Gründen, das Pferd erwerben will, obwohl der Tierarzt dessen Gesundheitszustand negativ beurteilt. Der Tierarzt tut in diesen Fällen gut daran, seine Einschätzung klar und deutlich kundzutun und festzuhalten. Auch in diesem Zusammenhang ist wieder auf die Gefährlichkeit von Prognosen über die künftige gesundheitliche Entwicklung eines Pferdes hinzuweisen.

Immer wieder kommt es vor, dass ein Käufer eine Kaufuntersuchung verlangt und der Verkäufer des Pferdes offeriert, diese vornehmen zu lassen und den Bericht vorzulegen. Der Verkäufer wird dann in der Regel den "Stalltierarzt" für diese Untersuchung beiziehen. In diesem Auftragsverhältnis Verkäufer-Tierarzt sind die Interessen der Vertragspartner nicht unbedingt gleich gerichtet. Je sorgfältiger der Tierarzł arbeitet, desto eher wird er nämlich die Chance des Verkäufers, das Pferd zu verkaufen, wegen der Entdeckung eines gesundheitlichen Mangels desselben vereiteln. Der Verkäufer als Auftraggeber des Tierarztes ist also nicht unbedingt an sorgfältiger Arbeit und an einem detaillierten Bericht interessiert. Deshalb ist diese Konstellation unserer Meinung nach sehr problematisch, vor allem aus der Sicht des Tierarztes.

Auch für den Pferdekäufer, dies sei hier nebenbei bemerkt, ist eine vom Verkäufer veranlasste Kaufuntersuchung wenig empfehlenswert. Zum einen wird sich die oben dargestellte Situation des Tierarztes eher zu seinen Ungunsten auswirken; 
zum andern entsteht zwischen ihm und dem untersuchenden Tierarzt kein Vertragsverhältnis, welches allenfalls die Grundlage für die Erhebung eines Haftungsanspruches gegen den Tierarzł abgeben könnte, wenn sich herausstellt, dass die Untersuchung unsorgfältig vorgenommen und deshalb ein gesundheitlich geschädigtes Pferd erworben wurde. Eine Rechtsnorm zum Schutze des Vertrauens des Pferdekäufers, der selbst nicht Vertragspartei des Tierarztes ist, im Sinne des deutschen Artikel 311 Abs. 3 BGB, kennt das schweizerische Recht nicht.

Prof. Reto Straub

Klinik für Nutztiere und Pferde

Universität Bern

Bremgartenstraße 106

CH 3012 Bern

reto.straub@knp.uni-be.ch nur für Gutachtertätigkeit gilt, nicht aber für Behandlungen.

Die Kaufuntersuchungen werden sowohl im Feld als auch in Privatkliniken und an der Vet Med Universität durchgeführt. Es soll darauf hingewiesen werden, dass besonders bei Untersuchungen im Feld schriftlich festgehalten werden muss, wenn auf Grund der Gegebenheiten, Teile der Untersuchung nicht durchgeführt werden können (zum Beispiel wenn kein ebener Boden vorhanden ist). Diese Pflicht ergibt sich unmittelbar aus dem $\$ 19$ Tierärztegesetz, wonach Zeugnisse und Gutachten nur nach gewissenhafter Erhebung und Untersuchung und unter genaver Beachtung der Regeln, Erkenntnisse und Erfahrungen der Veterinärmedizin nach bestem Wissen und Gewissen zu erstellen sind. Einige Kollegen gehen bereits aus Angst vor rechtlichen Konsequenzen dazu über, keine Kaufuntersuchungen mehr anzunehmen. Man kann nur hoffen, dass sich die gesetzliche Lage durch eine Gesetzesänderung oder eine eindeutige Rechtssprechung stabilisiert, sodass eine Rechtssicherheit wieder hergestellt ist.

\section{Vertragliche Haftungsbeschränkung des Tierarztes}

\section{Kaufuntersuchung und Pferdekauf in Österreich}

\author{
G. Hladik und M. Mosing
}

\section{Zur Kaufuntersuchung}

Bis zum heutigen Zeitpunkt wird der Verkauf vom Pferdehändler sehr unterschiedlich gehandhabt. Dies geht von detaillierten Verkaufsuntersuchungen bis zu reiner Übergabe des Papiers oder Pferdes gegen Geldübergabe. Auch bereits vor Eintritt des neven Gewährleistungsrechtes war ein verstärkter Trend zu tierärztlichen Kaufuntersuchungen bemerkbar.

Kaufuntersuchungen werden aufgrund unserer Erfahrung mit Pferden im Wert von 5.000 Euro aufwärts beauftragt, und derzeit meistens von Privatpersonen. Weiters sind Kaufuntersuchungen bei Pferden unter 3 Jahren eher die Ausnahme. In Österreich werden 3 Untersuchungen unterschieden:

Die Verkaufsuntersuchung - der Verkäufer gibt den Auftrag

Die Ankaufsuntersuchung - der Käufer gibt den Auftrag

Die Tiermängeluntersuchung - der Käufer gibt Auftrag nach dem Kauf

Gibt ein Verkäufer ein Verkaufsgutachten in Auftrag, so gilt das Gutachten auch als Beschreibung des Pferdes und somit als Vertragsbestandteil für den der Verkäufer einzustehen hat. Übernimmt der Tierarzt Beschreibung von Eigenschaften, kann ihn dies unter Umständen belasten: Beschreibt er z.B. ein M-Dressurpferd und dieses springt keine "Fliegenden Wechsel", so kann der Käufer sogar den Tierarzt belangen (Gutachtenvertrag mit Schutzwirkung zugunsten Dritter). Gegenüber dem Auftraggeber hat der Tierarzł für sein Gutachten nach dem Gewährleistungsrecht einzustehen. Hier sei betont, dass die Anwendbarkeit der Gewährleistungsregeln
Die Haftung des Tierarztes kann in Österreich weiterhin über die allgemeinen Vertragsbestimmungen geregelt werden. Einer ausdrücklichen privatrechtlichen Vereinbarung, wie in Deutschland vorgeschlagen, bedarf es in Österreich nicht. Allerdings ist zwischen Tierarzt und Konsument eine Haftungsbeschränkung - außer bei leichter Fahrlässigkeit - nich† möglich. Vorsatz lässł sich auch gegenüber dem Unternehmer nicht ausschließen. Grobe Fahrlässigkeit lässt sich nur bei Geschäften zwischen Tierarzt und Unternehmern ausschliessen. Die Veriährung der Ansprüche des Auftraggebers gegenüber dem Tierarzt aus dem Vertragsverhältnis kann von 3 Jahren auf sechs Monate verkürzt werden, jedoch nicht ab dem Tag der Untersuchung (wie dies in Deutschland der Fall ist), sondern erst ab Kenntnis des Schadens (Änderung der subjektiven Verjährungszeit). Weiters ist eine generelle Haftungsbeschränkung auf die Kaufpreissumme des Pferdes bei Verbrauchern - außer bei leichter Fahrlässigkeit - nicht möglich.

Eine große Zahl von Tierärzten benutz† vorgedruckte Formulare, die jedoch oft keine allgemeinen Vertragsbestimmungen enthalten. Zudem wird keine Unterschrift vom Auftraggeber gefordert, was für die Qualifikation als Urkunde wesentlich wäre. Mit der Unterschrift wäre die Echtheit der Urkunde vor Gericht von höherer Beweiskraft. Die allgemeinen Vertragsbedingungen können einen bedeutsamen Teil des Vertrages im Hinblick auf die rechtliche Absicherung darstellen: Es können bestimmte Haftungen und Risiken beschränkt bzw. ausGutachten an dritte Personen - und damit die Haftung gegenüber diesen - ausgeschlossen werden. Aufgrund der zum Teil offenen Auslegungsmöglichkeiten der neuen Gesetzeslage werden leider erst die ersten Prozessurteile richtungsweisend für die neve Gesetzeslage sein.

Allgemein kann gesagł werden, dass bezüglich der Klagefreudigkeit die Kaufuntersuchungen in Österreich bis jetzt eher einen geringen Teil der Gesamtklagen gegen Tierärzte darstellt. Aufgrund der verschärften und zum Teil unklaren Gesetzeslage dürfte sich das auch in Österreich bald ändern. geschlossen werden. Als Beispiel kann die Weitergabe von 
Bezüglich der schrifflichen Dokumentation der Befunde während der Untersuchung ergab eine Umfrage unter den österreichischen Pferdetierärzten, dass 95\% eine solche verwenden. In Österreich gibt es leider für die Untersuchung bis heute keinen einheitlichen Standard, sodass jeder Tierarzt nach seinem Ermessen höchst unterschiedliche Kaufuntersuchungsgänge und Protokolle verwendet. Da keine Standardisierung vorliegt, konnte festgestellt werden, dass einige Tierärzte sogar reine alternative Methoden, zum Beispiel Akupunktur bei Ihrer Kaufuntersuchung anwenden, was im Lichte der Lege-Artis-Untersuchungsverpflichtung nach dem Tierärztegesetz problematisch erscheint (§ 19 Tierärztegesetz). Ebenso existiert für die Bewertung der einzelnen erhobenen Befunde in Österreich kein Standard. Das heißt, dass zum Beispiel Beugeproben, die für den einen Tierarzt ein positives Ergebnis von einem Kreuz ergeben, für einen anderen drei Kreuz positiv sein können.

Diese unterschiedliche Bewertungen der Untersuchungsergebnisse führt leider auch in eine Grauzone bei der rechtlichen Beurteilung. Versuche in der Vergangenheit, eine Standardisierung von Befunden, wie z.B. der Vertragsvordruck der Pferdeheilkunde oder die von der Deutschen Röntgenkommission vorgeschlagenen Bewertungen einzuführen, finden bisher nur zum geringen Teil Beachtung. Eine Umfrage unter den Tierärzten ergab, dass der Wunsch nach Vereinheitlichung und somit besserer rechtlicher Absicherung besteht. Im Anschluss an diese Tagung soll versucht werden, in der Vereinigung der Österreichischen Pferdetierärzte (VÖP - www.pferdemedizin.at) eine solche Standardvereinbarung zu treffen.

Der Analyse der Kaufprotokolle der österreichischen Tierärzte wurde das Protokoll der Pferdeheilkunde als Basis zugrunde gelegt, da dies in Deutschland als Vorlage weit verbreitet ist. Folgende Punkte wurden von mehr als 30\% nicht angesprochen: Untersuchungsauftrag, Impfstatus, Endbewertung, Untersuchung unter dem Reiter, Untersuchung an der Longe, Wendeschmerz, Kaufpreisangabe und Vermögensschadensbegrenzung

Zusammenfassend kann gesagt werden, dass die schriftliche Untersuchungsniederlegung auch in Österreich gängige Praxis, die Gestaltung der Protokolle aber individuell verschieden und eine Rechtssicherheit dadurch nicht gegeben ist. Es sei erwähnt, dass es über die Verwendung von Protokollen und Vertragsvordrucken im tierärztlichen Bereich keine besonderen gesetzlichen Vorschriften gibt. Es besteht allerdings nach dem § 19/2 Tierärztegesetz die Verpflichtung, bei Gutachten und Zeugnissen, Aufzeichnungen zu führen und diese über 3 Jahre aufzubewahren.

\section{Gewährleistung beim Pferdekauf}

Das Tier wird zwar ausdrücklich im Gesetz vom rechtlichen Sachbegriff ausgenommen, aber das Tier wird als Sache behandelt, soweit keine Sonderbestimmungen für Tiere bestehen. Deshalb ist das Gewährleistungsrecht auch auf Pferde anzuwenden. Die Tatsache, dass ab 1.1.2002 ein neves Gewährleistungsrecht besteht, führte bis zum jetzigen Zeitpunkt in Österreich zu keiner spürbaren Reaktion der Vertragspartner. Weder die Tierärzte noch die Käufer und Verkäufer haben in erheblichem Maße auf die veränderte Lage reagiert.
Die wesentlichen Änderungen des neven Gewährleistungsrechtes wurden bereits an anderer Stelle ausführlich besprochen. Im Großen und Ganzen ist das österreichische Gewährleistungsrecht mit den Normen in Deutschland identisch. Folgende Unterschiede sind erkennbar:

\section{Beweislastumkehr}

Die Beweislastumkehr der allgemeinen Gewährleistung gilt in Österreich für jedes Geschäft und nicht, wie in Deutschland nur für Geschäfte zwischen Unternehmern und Verbrauchern (Verbrauchergeschäft). Das heißt, zum Beispiel beim Verkauf eines Pferdes zwischen 2 Privatpersonen hat in Deutschland im Falle eines Mangels der Käufer zu beweisen, dass der Mangel schon im Zeitpunkt der Übergabe vorhanden war. Im Gegensatz dazu hat in Österreich der Verkäufer die Beweislast für sechs Monate zu tragen und muss daher beweisen, dass der Mangel im Übergabezeitpunkt nicht vorhanden war. Die Beweislastumkehr kann allerdings vertraglich - außer bei Verbrauchergeschäften in obigem Sinne - ausgeschlossen werden, was aber aufgrund von Unkenntnis der Gesetzeslage sicher oft unterlassen wird.

\section{Mängel mit Vermutungsfrist}

Im Gegensatz zu Deutschland, wo alle Sonderregelungen über die sogenannten Hauptmängel mit der Schuldrechtsreform weggefallen sind, gelten in Österreich die sogenannten Viehmängel mit der Tiermängelverordnung weiterhin. Grundsätzlich gilt die Tiermängelverordnung, die Vermutungsfristen für bestimmte Mängel festgelegt, für alle Tiere. Die Verordnung besagt beim Pferd dass innerhalb einer Frist von 7 - 14 Tagen je nach Mangel, der Käufer diesen zu melden hat und der Verkäufer beweisen müsste, dass dieser im Zeitpunkt des Kaufes nicht vorhanden war.

Die Tiermängelverordnung ist nunmehr nach dem Konsumentenschutzgesetz in Österreich bei Verbrauchergeschäften nicht mehr anwendbar, weil ja ohnedies die allgemeine Beweislastumkehr von 6 Monaten vorliegen würde. Bei reinen Konsumenten oder reinen Unternehmergeschäften gilt die Verordnung, soweit sie vertraglich nicht ausgeschlossen wird, weiterhin.

Folgende Problematik ist nun bei der neven Gesetzeslage beim Verbrauchergeschäft zu beachten: Die 6-monatige Beweislastumkehr wurde von der österreichischen Lehre ( Jud und Welser, die auch an der Gesetzeswerdung beteiligt waren) dahingehend eingeschränkt, dass Tierkrankheiten nicht 6 Monate rückdatierbar sind. Da nun - wie oben dargestellt - die Tiermängelverordnung beim Verbrauchergeschäft seit Inkrafttreten des neven Gewährleistungsgesetzes nicht anwendbar ist, entsteht für den Konsumenten eine Verschlechterung, weil er in jedem Fall bei Krankheiten die Beweislast zu tragen hätte. Hat zum Beispiel ein Pferd einen Mangel, wie etwa periodische Augenentzündung, wäre nach der TMV bei Meldung innerhalb von 7 Tagen der Verkäufer verpflichtet den Beweis anzutreten. Nach dem neven Gewährleistungsgesetz wäre allerdings der Verkäufer sowieso verpflichtet in den ersten 6 Monaten zu beweisen, dass der Mangel bei Übergabe nicht vorhanden war. 
Es entsteht ein logischer Widerspruch, weil nach der österreichischen Lehre die 6 Monatsfrist bei Tierkrankheiten allgemein nicht anwendbar ist und nach dem Gesetz die Tiermängelverordnung bei Verbrauchergeschäften nicht gilt. Daraus ergeben sich in den kommenden Gerichtsverfahren zwei Reaktionsmöglichkeiten: Entweder wird die TMV sinngemäß beim Konsumentengeschäft doch angewendet, oder es gilt die allgemeine 6 monatige Beweislastumkehr auch für Tierkrankheiten. Wie genau verfahren wird, werden die ersten Gerichtsurteile zeigen.

Die Viehmängelfrist von sechs Wochen zur Geltendmachung der Mängel vor Gericht ist nach der Rechtsprechung nur für landwirtschaftliche Nutztiere, also nicht für Luxustiere wie ein Sportpferd, anwendbar. Für Sportpferde gilt somit eine Geltendmachungsfrist von nunmehr zwei Jahren. Diese Frist kann gegenüber einem anderen Unternehmer oder zwischen zwei Konsumenten eingeschränkt oder ganz ausgeschlossen werden. Hingegen ist sie bei Verbrauchergeschäften unter bestimmten Umständen (gebrauchte Sachen) durch ausdrückliche Vereinbarung auf ein Jahr verkürzbar.

\section{Gewährleistung}

Weiters gibt es in Österreich keine Sonderregelungen für Auktionen. In Deutschland gelten in diesem Fall keine 6 Monate Beweislastumkehr. Die Umsetzung der Gewährleistungsansprüche gestaltet sich im Falle des Pferdekaufs schwierig. Da es sich um ein Lebewesen handelt, sind die primären Behelfe des GLR, nämlich Austausch oder Reparatur oft nicht möglich, wie es z. B. bei einer Waschmaschine der Fall wäre. Somit sind die sekundären Gewährleistungsbehelfe, nämlich Preisminderung oder Wandlung beim Pferdekauf die häufigsten Ansprüche im Mängelfall.

Dr. Georg Hladik

Pferdeklinik Pegasus

Laaber Straße

A 2384 Breitenfurt

pferdeklinik.pegasus@nextra.at

\section{Das neue Pferdekaufrecht}

\author{
J. Adolphsen
}

Europa hat uns zum Jahreswechsel nicht nur neues Geld beschert, sondern auch neve Regeln, wie wir dieses Geld beim Kauf einsetzen können. In der Vorweihnachtszeit warben große Handelsketten bereits damit, den Kunden im Vorgriff auf das neue Recht die ab 2002 geltende Gewährleistung zu bieten. Diese Rechtsänderung ist es, die auch Einfluss auf den Kauf von Pferden hat.

Mit der Reform des Schuldrechts - und hier in erster Linie des Kaufrechts - , die der deutsche Gesetzgeber aufgrund der europäischen Verbrauchsgüterkaufrichtlinie zum Jahreswechsel vorgenommen hat, entfallen die bisherigen Spezialregelungen für den Pferdekauf. Es gibt keine Gewährsmängel mehr, keine Gewährsfristen und auch keine gegenüber dem normalen Kauf von Sachen abweichenden Verjährungsregeln. Der Wegfall dieser Regeln erfolgt zu Recht, weil sie der Entwicklung des Pferdes vom landwirtschaftlichen Nutztier zu einem Hochleistungssportpferd weit hinterher hinkten und einseitig die Verkäufer begünstigten. Ob aber die neven gesetzlichen Regeln "besser" sind, muss zum jetzigen Zeitpunkt jedenfalls skeptisch beurteilt werden, weil das Kaufrecht ersichtlich nicht für Tierkäufe gedacht ist, sondern den Kauf beweglicher Sachen wie Autos oder Toaster im Auge hatte. Trotzdem bietet das Gesetz einige begrüßenswerte Neuerun- gen und zudem begrenzte Möglichkeiten vertraglicher Gestaltung, die, wenn die Rechtsprechung diese Vertragsgestaltungen billigte, zu einer handhabbaren Regelung führen könnten.

\section{Verbrauchsgüterkauf und sonstiger Pferdekauf}

Das neue Recht zwingt dazu, Verbrauchsgüterkäufe von sonstigen Pferdekäufen zu unterscheiden. Der Unterschied zwischen beiden liegt in der Beteiligung eines Verbrauchers und eines Unternehmers. Verbraucher ( $(13$ BGB) ist jeder, der den Vertrag zu einem Zweck abschließt, der nicht zu seiner gewerblichen oder selbständigen beruflichen Tätigkeit zählt, also der Hobby (Turnier-) reiter. Unternehmer ( $§ 14$ BGB) ist jemand, der den Vertrag in Ausübung seiner gewerblichen oder selbständigen beruflichen Tätigkeit abschließt, also der Pferdehändler aber auch der Bereiter etc.

Verbrauchsgüterkäufe sind ausschließlich Käufe eines Verbrauchers von einem Unternehmer, also nicht Verträge zwischen Verbrauchern, Verträge zwischen Unternehmern oder der Kauf des Unternehmers von einem Verbraucher! Verbrauchsgut ist, das mag einem gefallen oder nicht, ein Pferd immer dann, wenn es ein Verbraucher von einem Unternehmer erwirbt.

Die Unterscheidung von Verbrauchsgüterkäufen und sonstigen Pferdekäufen ist aus zwei Gründen wichtig: erstens unterscheiden sich die gesetzlich geltenden Regeln für Verbrauchsgüterkäufe und sonstige Käufe und zweitens differieren die Möglichkeiten vertraglicher Gestaltung. 


\section{Der Mangelbegriff}

Im Zentrum der Rechtsänderung steht ein never Mangelbegriff. Bisher gab es, wenn nichts anderes vereinbart wurde, beim Viehkauf nur sechs vom Gesetz als erheblich angesehene Mängel, die ehem. Gewährsmangel. Heute haben es die Parteien in der Hand festzulegen, was ein Mangel ist, indem sie eine Vereinbarung über den Sollzustand treffen. Dazu enthält das Gesetz eine Dreistufenregelung (§ 434 Abs. 1 BGB): Ein Pferd ist mangelfrei

1. wenn es bei Vertragsschluss die vereinbarte Beschaffenheit hat. Mangels Vereinbarung ist es frei von Mängeln,

2. wenn es sich für die nach dem Vertrag vorausgesetzte Verwendung eignet oder

3. wenn es sich für die gewöhnliche Verwendung eignet und eine Beschaffenheit aufweist, die bei Tieren gleicher Art üblich ist und die der Käufer nach der Art des Tieres erwarten kann.

Es empfiehlt sich in Zukunft unbedingt, die vereinbarte Beschaffenheit in einem schriftlichen Vertrag umfassend festzuhalten, um nicht in Stufe zwei bzw. drei zu geraten, denn es ist ausgesprochen schwierig, im Streitfall eine vorausgesetzte Verwendung bzw. die Eignung für eine gewöhnliche Verwendung gerade bei Sportpferden zu bestimmen. Eine Vereinbarung der Beschaffenheit eines Pferdes im Sinne der Stufe 1 ist aber deutlich schwieriger als die Vereinbarung der Beschaffenheit eines Autos. Ein Auto soll eine bestimmte Leistung erbringen, was man anhand objektiv messbarer Daten bestimmen kann. Ein Auto erbringt diese Leistung auch mit dem Käufer. Bei einem Pferd muss sich die Vereinbarung auf den Gesundheitszustand und den Ausbildungsstand erstrekken.

\section{Vereinbarung über den Gesundheitszustand}

Eine durchzuführende tierärztliche Untersuchung muss in Zukunft Vertragsinhalt werden und zwar in der Form, dass der Vertrag nicht zustande kommt, wenn das Pferd die Untersuchung nicht passiert (sog. aufschiebende Bedingung). Das Ergebnis der Untersuchung wird Vertragsinhalt. Soweit dabei Veränderungen des Gesundheitszustandes aufgedeckt werden und der Käufer das Pferd trotzdem kauft, hat er keine Rechte bezüglich der aufgedeckten Mängel (§ 442 BGB). Je umfangreicher also die Untersuchung ist, desto leichter kann ein Streit entschieden werden. Den Umfang der Untersuchung müssen die Parteien miteinander vereinbaren, dabei ist der Rat eines Tierarztes notwendig. Werden später Normabweichungen innerhalb oder außerhalb des Untersuchungsumfangs festgestellt, so ist anhand der dargestellten Stufen 2 bzw. 3 zu prüfen, ob ein Mangel vorliegt, der dem Käufer Rechte gegenüber dem Verkäufer gewährt. Die Vereinbarung einer tierärztlichen Untersuchung kann also letztlich nicht verhindern, dass man in die problematischen Stufen 2 und 3 gerät.

\section{Vereinbarung über den Ausbildungsstand}

Zusätzlich sollten die Parteien eine Vereinbarung über den aktuellen Ausbildungsstand treffen. Hierzu sind objektiv nachweisbare Daten aus der Vergangenheit zu verwenden werden,
z.B. Platzierungen und Turniereinsätze. $\mathrm{Zu}$ vermeiden sind unbedingt Angaben, die irgendwie in die Zukunft reichen, also Aussagen wie: "Das Pferd ist in M-Springen einsetzbar." Niemand weiß, ob das Pferd mit dem neuen Käufer auch über M springt! Vorsicht ist in Zukunft geboten bei werbeartigen Aussagen über die Eignung des Pferdes, weil diese als Teil der Vereinbarung gelten können und darauf Mängelrechte gestütz† werden können.

\section{Rechte im Fall eines Mangels}

Tritt ein Mangel nach Vertragsschluss auf, kann der Käufer nicht wie bisher sofort vom Kaufvertrag loskommen. Er muss erst Nacherfüllung, d.h. nach seiner Wahl die Beseitigung des Mangels oder eine Ersatzlieferung verlangen (§ 437 Ziff. 1 BGB). Die Lösung vom Vertrag durch Rücktritt (Pferd zurück, Geld zurück) oder die Minderung (§ 441 BGB, Anpassung des Kaufpreises an den realen Wert) sind grundsätzlich erst möglich, wenn die Nacherfüllung gescheitert ist. Insofern besteht an sich ein klares Stufenverhältnis: erst Stufe 1, wenn das nicht klappt Stufe 2.

Diese Regeln sind ersichtlich für Käufe über Sachen gemacht, die als Massenware angeboten werden, wie eben Autos, Elektrogeräte etc., nicht aber für Tiere. Bei diesen ist eine Mängelbeseitigung oft unmöglich (chronische Krankheiten). Allerdings kann die Rückgabe eines Pferdes zum Auskurieren eines Hustens, zur Verbesserung des Futterzustandes oder auch zur Beseitigung von Ausbildungsmängeln erwogen werden. Eine Ersatzlieferung scheitert meist schon daran, dass es das betreffende Tier nur einmal gibt (sog. unvertretbare Sache). Die Rechte der Stufe 1 wählen zu müssen, kann darüberhinaus für den Käufer unzumutbar sein, vor allem, wenn ihm sein neves Pferd schon ans Herz gewachsen ist.

Die Minderung ist ein für Viehkäufe never Rechtsbehelf, der bisher von vielen deshalb als für den Pferdekauf ungeeignet gehalten wurde, weil es für Pferde keine festen Marktpreise gebe. Allerdings trifft dies für viele Kaufgegenstände, wie z.B. Kunstwerke zu, für die aber immer schon ein Minderungsrecht bestand. Der Käufer kann darüber hinaus bei Lieferung eines mangelhaften Pferdes Ersatz für Schäden verlangen, die ihm durch die Mangelhaftigkeit des Pferdes entstehen ( $\S \S 280$, 281 BGB, z.B. Behandlungskosten beim Tierarzt), soweit der Verkäufer nicht nachweist, dass er den Mangel nicht zu vertreten hat.

\section{Beweislast}

Die Beweislast ist im Prozess dann von Bedeutung, wenn sich eine Tatsache, auf die es für die Entscheidung ankommt, nicht beweisen lässt, z.B. die Frage, ob ein Chip bereits bei Vertragsschluss vorhanden war. Dann kommt es darauf an, zu wessen Lasten die Unaufklärbarkeit geht.

Grundsätzlich muss der Käufer beweisen, dass ein Mangel besteht und dass dieser bei Vertragsschluss bereits vorlag. Kann er das nicht, weil es möglich ist, dass der Mangel nach Vetragsschluss aufgetreten ist, wird die Klage als unbegründet abgewiesen.

Das Gesetz bringt eine nur für den Verbrauchsgüterkauf geltende Neuerung (§ 476 BGB): der Verkäufer muss sechs 
Monate lang beweisen, dass ein Mangel, der in dieser Zeit auftritt, nicht schon bei Vertragsschluss vorlag. Außerhalb dieser Frist und außerhalb des Verbrauchsgüterkaufs gilt diese Beweislastumkehr nicht!

Diese Beweislastumkehr in der Zeit von sechs Monaten ist für Pferdekäufe ungeeignet. Sie gilt nicht, wenn dies mit der Art des Sache oder des Mangels nicht vereinbar ist. Da sich der Zustand eines Tieres, (abhängig von Haltung, Pflege und Belastung) in sechs Monaten erheblich verändert, spricht vieles dafür, dass die Beweislastumkehr in ihrer konkreten Erscheinung beim Tierkauf mit der Art des Kaufgegenstandes Tier generell unvereinbar ist. Der Gesetzgeber hat es im Gesetzgebungsverfahren leider abgelehnt, ausdrücklich anzuordnen, dass dies bei Tieren grundsätzlich der Fall ist.

\section{Verjährung}

Ist ein Anspruch veriährt, kann er auf Einrede des Gegners nicht mehr gerichtlich geltend gemacht werden; eine Klage würde als unbegründet abgewiesen. Die regelmäßige Veriährung der Rechte aus einem Kaufvertrag über ein Pferd beträgt zwei Jahre nach der Ablieferung (§ 438 Abs. 1 Nr. 3 BGB). Der Gesetzgeber hält diese Regelung auch bei Tierkäufen für angemessen, womit er jedoch falsch liegt. Die Frist ist für Tierkäufe völlig ungeeignet, innerhalb eines derart langen Zeitraums werden Prozesse zum reinen Vabanquespiel.

\section{Vertragliche Gestaltungen}

In der Zukunft ist es unbedingt nötig, die gesetzlichen Regeln durch Vertrag so abzuändern, dass eine Vertragsgestaltung erfolgt, die den Interessen beider Parteien gerecht wird. Diese Möglichkeiten sind jedoch nicht unbegrenzt. Die Grenzen werden vom Gesetz gebildet durch Regeln, die der Gesetzgeber im Kaufrecht als nicht abdingbar gekennzeichnet hat und durch die Regeln, die für die Gestaltung von Verträgen durch Allgemeine Geschäftsbedingungen („das Kleingedruckte") gelten ( $\S \S 305$ ff. BGB).

Wenn jemand ein Vertragsmuster verwendet (auch wenn es von einem Verband wie der FN oder einem Rechtsanwalt erstellt ist) gelten in jedem Fall die Regeln über Allgemeine Geschäftsbedingungen. Wenn ein Verkäufer Unternehmer ist und an einen Verbraucher verkauft, sind die Regeln des Verbrauchsgüterkaufs zwingend. Wer als Unternehmer an einen Verbraucher verkauft und einen Vertrag bzw. einen Mustervertrag für eine Vielzahl von Verträgen nutzt, gelten beide Grenzen.

Zu einer Vertragsgestaltung ist der Rat eines kompetenten Anwalts erforderlich, der auch über die Grenzen und Risiken vertraglicher Regelungen aufklären wird.

Zur Zeit fehlt naturgemäß noch jede Rechtsprechung zum neuen Recht, so dass Aussagen, dass bestimmte Regelungen sicher "halten", mit Vorsicht zu genießen sind. Im letzten Jahr sind bereits Vertragsmuster erschienen, deren Verfasser diese Grenzen auf jeden Fall nicht beachtet haben!

\section{Verbrauchsgüterkaufverträge}

Mangel

Je weiter die Vereinbarung über die Beschaffenheit des Pferdes reicht, desto leichter kann ein Streit entschieden werden. Der Verkäufer hat ein Interesse daran, dass der Käufer viele Fehler des Pferdes kennt. Dies klingt natürlich widersprüchlich, erklärt sich aber dadurch, dass der Käufer keine Rechte bei Kenntnis eines Mangels hat.

Rechte im Falle eines Mangels

Eine Vereinbarung über die Rechte im Falle eines Mangels zu Lasten des Verbrauchers, ist bei Verbrauchsgüterkäufen unzulässig. Ein Haftungsausschluss (gekauft wie besichtigt etc.) ist deshalb nicht möglich.

Beweislast

Die Regel über die Beweislastumkehr sind im Verbrauchsgüterkauf nicht abdingbar. Spätestens im Prozess stellt sich aber die Frage, ob eine Beweislastumkehr über sechs Monate mit der Art des Kaufgegenstandes Pferd oder der Art des Mangels unvereinbar ist, so dass die durch Gesetz angeordnete Beweislastumkehr schon nicht gilt. Hierfür spricht einiges, so dass man durchaus den Versuch machen kann, die Beweislast des Käufers durch Vertrag festzuschreiben. Die Rechtsprechung wird in der Zukunft diese Frage zu entscheiden haben.

Verjährung

Die Veriährung der Rechte im Fall eines Mangels kann vor Mitteilung des Mangels an den Unternehmer nicht unter zwei Jahre vertraglich verkürzt werden. Bei gebrauchten Sachen ist es möglich, die Frist auf ein Jahr zu verkürzen (§ 475 Abs. 2 BGB). Bei Pferden spricht einiges dafür, diese immer wie eine gebrauchte Sache zu behandeln, weil man ansonsten in Abhängigkeit von der vorgesehenen Verwendung prüfen müsste, ab welchem Zeitpunkt ein Pferd als gebraucht anzusehen ist. Dies dürfte zu skurilen Ergebnissen führen. Insofern könnte sich eine Verkürzung der Frist auf ein Jahr empfehlen. $\mathrm{Ob}$ dieses Vorgehen einer gerichtlichen Prüfung standhält, ist zur Zeit offen.

\section{Sonstige Kaufverträge}

Hier gelten die strengen Regeln des Verbrauchsgüterkaufs ( $\S$ $474 \mathrm{ff}$. BGB) nicht, so dass Vereinbarungen in weiterem Umfang zulässig sind. Die Grenzen der Regeln über Allgemeine Geschäftsbedingungen ( $\S \S 305$ ff. BGB) sind auch hier zu beachten.

\section{Haftungsausschluss}

Ein Haftungsausschluss in Einzelverträgen ist zulässig. Ob ein gänzlicher Haftungsausschluss in Musterverträgen oder sonstigen vorformulierten Verträgen möglich ist, ist deshalb zweifelhaft, weil die Regeln über Allgemeine Geschäftsbedingun- 
gen dies jedenfalls bei neven Sachen verbieten ( $§ 309$ Nr. 8 $b$ aa $B G B)$. Also hängt die Zulässigkeit des Haftungsausschlusses an der Einordnung des Pferdes als gebrauchte Sache. Versuchen kann man eine solche vertragliche Gestaltung, ob sie einer gerichtlichen Prüfung standhält, kann niemand zur Zeit sicher sagen, obgleich viel dafür spricht.

Verjährung

Für sonstige Pferdekäufe kann die Länge der Veriährungsfrist frei ausgehandelt werden.

In Musterverträgen kann die Veriährung dann nicht unter ein Jahr verkürzł werden, wenn man das Pferd als neve Sache ansieht (§ $309 \mathrm{Nr}$. 8b ff BGB). Ordnet man es dagegen als gebrauchte Sache ein, so kann die Veriährungsfrist frei vereinbart werden.

\section{Schluss}

Für sonstige Pferdekaufverträge bieten sich vertragliche Gestaltungen an, die auch das neve Recht den jeweiligen Bedürfnissen der Parteien anpassen können. Für Verbrauchs- güterkaufverträge trifft dies dagegen nicht zu: hier haben sich die Vorzeichen des Pferdekaufs vollkommen verschoben, da nun die Käufer, die 100 Jahre kaum Rechte im Falle eines Mangels hatten, plötzlich alle Trümpfe in der Hand halten. Die aufgezeigten Grenzen müssen von den Parteien, vor allem von den Verkäufern, die ihre Haftung in Grenzen halten wollen, eingehalten werden. Ein Unternehmer, der ein Pferd an einen Verbraucher verkaufen will, muss heute selbst dafür sorgen, dass das Pferd von einem kompetenten Tierarz† umfassend untersucht wird. Wer als Verkäufer ein Pferd ohne Mängel zu offenbaren oder durch eine Untersuchung aufdecken zu lassen, vom Hof gibt, setzt sich der Gefahr aus, dass der Käufer noch mindestens ein Jahr nach der Übergabe des Pferdes Rechte geltend macht. Vorsicht geboten ist hier vor allem vor Minderungsverlangen, die in Zukunft auch rechtsmissbräuchlich eingesetz† werden können.

Dr. Jens Adolphsen

Universität Regensburg

jens.adolphsen@jura.uni-regensburg.de

\section{Die Augenuntersuchung im Rahmen von Kaufuntersuchungen bei Pferden: Vorschlag für eine Standardisierung des Untersuchungs- protokolls}

\author{
H. Gerhards
}

Chirurgische Tierklinik, Ludwig-Maximilians-Universität München

\section{Einleitung}

Die Augenuntersuchung war und ist Bestandteil jeder tierärztlichen Kaufuntersuchung bei Pferden. Nach dem Wegfall der "Kaiserlichen Verordnung" gibt es bezüglich der Augenuntersuchung keine einschränkende Vorgabe für den Untersuchungsauftrag mehr, wie sie vorher bei dem Auftrag "Untersuchung auf den Hauptmangel periodische Augenentzündung" noch hin und wieder vorkommen konnte. Vielmehr muss nun nach $\S 434$ BGB n.F. auf "Sachmängelfreiheit" untersucht werden. Neben den rechtlichen Aspekten und Vorgaben wird es zumindest bei sportlich genutzten Pferden im mutmaßlichen Interesse eines Erwerbers liegen, ein Pferd mit gesunden Augen, d.h. mit "normaler" Sehfähigkeit und ohne gravierende kosmetische Beeinträchtigungen, wie sich etwa aus häßlichen Sekretstraßen, entstellenden Lidverletzungen und Augapfelschrumpfungen ergeben können, zu übernehmen. Einem Züchter dürfte darüber hinaus wichtig sein, vor dem Erwerb eines zur Zucht bestimmten Pferdes das Vorliegen kongenitaler Anomalien und Missbildungen der Augen und ihrer Adnexe, deren Erblichkeit nicht ausgeschlossen werden kann, auszuschließen.

\section{Umfang der Augenuntersuchung bei Kaufuntersuchun- gen}

Aus anatomischen und physiologischen Gründen kann sich die Augenuntersuchung nicht nur auf das eigentliche Sehorgan beschränken, sondern muss die Untersuchung der Augenadnexe einbeziehen, da Augenadnexe und Augapfel eine funktionelle Einheit bilden. Das bedeutet, dass im - zwekkmäßigerweise eine anatomische Reihenfolge einhaltenden Untersuchungsprotokoll folgende Strukturen einer Adspektion und oder Palpation (knöcherne Orbita, ggf. Augäpfel) unterzogen werden müssen:

- Umgebung der Augen inkl. knöcherne Orbita

- Augenlider inkl. Lidränder und Zilien

- Konjunktiva palpebrae et bulbi

- Tränenpünktchen (nur bei chronischer Tränenspur bzw. im Verdachtsfall) 
- Mündungen der Tränenkanäle im Nasenvorhof (Vorhanden sein, Sekretion)

- Palpebra tertia (Lateralfläche)

- Bulbi oculi (Größe, Position, ggf. i.o. Druck, Prüfung durch transpalpebrale Palpation)

- Sklera

- Kornea

- vordere Augenkammer

- Iris

- Pupillenreaktion

- Linse

- Glaskörper

- Fundus inkl. Papilla nervi optici und peripapilläre Gefäße

- Reaktion des Pferdes auf die in

Es gilt kongenitale, traumatische wie auch entzündliche und tumoröse Veränderungen der Augen und ihrer Adnexe festzustellen bzw. deren Vorliegen auszuschließen. Weiter muss die Sehfähigkeit des Pferdes anhand der in der Veterinärophthalmologie gebräuchlichen Tests überprüft und beurteilt werden, ob und ggf. inwieweit vorliegende Veränderungen die Sehfähigkeit und die Gebrauchsfähigkeit beeinträchtigen. In manchen Fällen dürfte es außerdem auf eine prognostische Beurteilung eines Befundes ankommen.

Diesen Pflichten kann nur durch eine gründliche und vollständige, klinische und ophthalmologische Untersuchung Genüge getan werden. Voraussetzungen hierfür sind eingehende Kenntnisse der normalen und pathologischen Befunde an Augenadnexen und Augen, eine geeignete Umgebung (ruhiger Raum, helles diffuses Licht wie auch eine Möglichkeit zur Untersuchung im Dunkelraum), Verfügbarkeit von für Pferde geeignete Augenuntersuchungsinstrumenten (helles fokales Licht mit großem Leuchtfleck, Kopfband-Lupe, Ophthalmoskop, Spaltlampe, Lidhalter), Diagnostika (kurzwirkendes Mydriatikum, Lokalanästhetikum, Fluorescein).

Hier alle pathologischen Befunde an den Augen und ihren Adnexen im Einzelnen aufzuzählen und zu bewerten würde den Rahmen einer Veröffentlichung sprengen. Vielmehr wird auf einschlägige Lehrbücher und Einzelpublikationen zu Augenkrankheiten bei Pferden verwiesen, wobei als Referenz und Diagnostikhilfe der Bildatlas von Barnett und Mitarbeitern besonders dienlich ist.

Selbstverständlich kann es im Zuge einer Kaufuntersuchung nur darum gehen, Unsicherheiten in der Befundbeurteilung, die sich bei der klinischen und ophthalmologischen Untersuchung ergeben, durch einfache, nicht invasive Untersuchungen zu ergänzen. So kann und sollte bei entsprechendem Verdacht, der sich aufgrund adspektorisch erhobener Befunde (Mündungen der Tränenkanäle ohne Sekrete) ergeben haben könnte, die Durchgängigkeit des Tränenkanals durch Einträufeln von Fluorescein in den Konjunktivalsack und Kontrolle der Mündung des Tränenkanals überprüft werden. Hingegen sind Sondierung und/oder Spülung des Tränenkanals nicht selbstverständlicher Bestandteil der Kaufuntersuchung sein. Andererseits kann nur dann auf die medikamentöse Pupillenerweiterung verzichtet werden, wenn die Pupillenweite ausreicht, die Strukturen des Augeninneren eingehend zu ophthalmoskopieren. In allen anderen Fällen sollte zumindest auf die Möglichkeit der medikamentösen Pupillenerweiterung und die Folgen deren Unterlassung (unvollständige Untersu- chung der inneren Augenstrukturen) hingewiesen werden. Lidhalter und Anästhetikum dienen im Rahmen einer Kaufuntersuchung nur dazu, verdächtige oder unklare Befunde z.B. durch Anheben eines Lides der besseren Betrachtung zugänglich zu machen. Schließlich ist der Untersucher verpflichtet, in Verdachtsfällen und in Zweifelsfällen auf die Möglichkeiten weitergehender Untersuchungen hinzuweisen (Spaltlampenuntersuchung, intraokulare Druckmessung, Sonographie, ERG,....)

\section{Literatur}

Barnett, K. C., S. M. Crispin, J. D. Lavach und A. G. Matthews (1998): Augenkrankheiten beim Pferd. Farbatlas für die Praxis. Schlütersche Verlag und Druckerei, Hannover

Parker, J. und D. J. Habin (1994/1995): Examination of the equine eye as part of the prior to purchase examination: Part I, II and III. Equine vet. Educ. 6, 333ff., 7, 43-48, u. 94-98

Prof. Dr. H. Gerhards

Pferdeabteilung der Chirurgischen Tierklinik

Universität München,

Veterinärstr. 13

D-80539 München

h.gerhards@chir.vetmed.uni-muenchen.de

\section{Neurologische Untersuchung im Rahmen der Kaufuntersuchung}

\author{
R. Straub R. und Heidrun Gehlen \\ Klinik für Nutztiere und Pferde, Universiotät Bern
}

Die neurologische Untersuchung ist ein integraler Bestandteil einer korrekt durchgeführten und vollständigen inneren Ankaufs- oder Verkaufsuntersuchung. Die Methoden der Untersuchung zum Aufdecken subklinisch verlaufender Störungen oder Erkrankungen mit schwacher neurologischer Symptomatik sind beim Pferd beschränkt. Die zur Verfügung stehenden Methoden zur Prüfung von zentral- oder periphernervösen Störungen sind einfach und grobsinnlich, die Interpretetion der Resultate aufgrund der mangelnden Standardisierungen oft zu subjektiv. Auch ergänzende Untersuchungsmethoden der bildgebenden Verfahren sind dementsprechend schwierig zu beurteilen.

Im Rahmen der Ankaufs- oder Verkaufsuntersuchung ist am ruhigen, stehenden Pferd in vertrauter Umgebung folgendes zu untersuchen, zu beurteilen resp. auszuschließen:

- Allgemeines Verhalten

- Haltung (Kopf-, Hals- und Körperhaltung)

- Symmetrie (Kopf, Hals, Körper, Gliedmassen) 
- Reflexverhalten (Ohr-,Augenlid-, Pupillen-, Stirn-, Fliegen-, Perianal-, Vulvareflex)

- Sensibilität (Oberflächen- und Tiefensensibilität)

- Muskeltonus (Hals-, Rücken-, Zungen- und Schweifmusku latur)

- Halsmotilität (aktiv und passiv)

- Hautuntersuchung (Neurektomie- und Sturznarben; Schwit zen)

- Hintergliedmassen ( beim Aufhalten)

- Luftsack (Sek. Nervenaffektion)

- Augenuntersuchung

- Motorik: Schritt und Trab (frei oder an der Hand); gerade aus und auf dem Zirkel,

- auf hartem und weichem Boden; bergauf und bergab.

- Rückwärtstreten (an der Hand oder unter dem Reiter).

- Belastungsprobe (Atemgeräusche und Rittigkeit)

Nachfolgend wird der Ablauf unseres Untersuchungsganges vorgestellt. Beispiele potentiell subklinisch verlaufender Erkrankungen und Symptome werden zu den Untersuchungssequenzen in Klammern gesetzt.

Die Beurteilung des Verhaltens unter Berücksichtigung von Konstitution und Temperament desjenigen Pferdetypes (Apathie: hochzentrale Affektion; Schmerzen: Rückensyndrom u.a.; Übererregbarkeit: hochzentrale Symptomatik; Hyperthyreose) ist sehr heikel und erfordert viel Erfahrung und Einfühlungsvermögen. Dies umsomehr, als bei den meisten Ankaufsuntersuchungen weder der Mandant noch der Tierarzt mit dem Verhalten des zu untersuchenden Tieres vertraut ist. Im Wesentlichen gilt es für den untersuchenden Tierarzł neben Apathie oder Übererregbarkeit, Stereotypien (Koppen, Zungenspielen, Lippenschlagen, Scharren, Weben, Boxenlaufen, Schweifschevern: Stereotypie aber auch Folge einer Paraesthesie bei Cauda equina Syndrom; u.a.) und Hyperkinesien (Kopftick; Intensionstremor: Kleinhirnerkrankung) auszuschliessen. Asymmetrien, hervorgerufen durch Muskelatrophie und/oder

Muskellähmungen werden oft mit primären oder sekundären Nervenaffektionen werden in Zusammenhang gebracht werden (Facialisparese; Horner Syndrom Kehlkopfasymmetrie: Hemiparesis laryngis; Wurzelneuritis: Rückensyndrom; Lähmungen peripherer Nerven wie z.B der Nn. suprascapularis, radialis, fibularis und tibialis haben meist eine offensichtliche Symptomatik). Auch Lageveränderungen des Beckens und Auftreibungen im Bereich knöcherner Strukturen weisen möglicherweise auf sekundäre Affektionen des Nervensystem hin. Die Kopf- (Vestibulärsyndrom, Blindheit),Hals-(Cervicalsyndrom), Körper- und Gliedmassenstellung (EMND; EDM) und das Reflexverhalten sind wiederholt zu prüfen und vorsichtig zu interpretieren. Drohreflex (Affektion des N.opticus), Augenlidreflex (Affektion des N.trigeminus und N.facialis), Pupillarreflex (Affektion des N.opticus und N. occulomotorius), Schnippenreflex (Affektion des N.trigeminus; Auslösen eines Kopftics), Panniculusreflex (Hyper-, Hypo- und Anaesthesien), Perianal- und Vulvareflex (Cauda equina Syndrom). Das Prüfen und die Beurteilung der Oberflächensensibilität, im Speziellen der distalen Gliedmassen (erfolgreiche Neurektomie) ist unproblematisch, jedoch von grosser forensischer Bedeutung. Die Interpretation des Kreuztestes (Störung der Tiefensensibilität) bei träger Stellungskorrektur ist oft schwierig. Im Weiteren ist die Beurteilung des Muskeltonus der Hals- und Rückenmuskulatur mit und ohne Druck auf die Akupunktur- oder Stresspunkte und die Meridianlinie von Bedeutung (Zervikal- und Rückensyndrom). Ein herabgesetzter Zungentonus muss nicht (Zungenspieler) aber kann Hinweise auf eine Innervationsstörung liefern (Affektion des N. hypoglossus und N. glossopharyngeus; (Luftsackmykose) ebenso ein herabgesetzter Schweiftonus (Cauda equina Syndrom; cave! an Schweifriemen gewohnte Equiden). Wertvoll ist auch die ergänzende Prüfung der aktiven und passiven Hals- und Rückenmotilität und Sensibilität (Zervikal- resp. Rückensyndrom). Man tut gut daran spärlichen Nasenausfluss genau zu beurteilen (Luftsackmykose). Die Haut ist auf Narben abzusuchen (Neurektomie- und Sturznarben (Narkolepsie, Asynergien; lokalkes Schwitzen: lokale Sympathikusaffektion). Das Aufhalten der Gliedmassen (Hyperkinesien: Shivering) verrät oft Bewegungsstörungen. Zum Schluss gehört die Augenuntersuchung, insbesondere die der Cornea (mangelhafter Lidschluss: zentrale Affektionen des N.facialis ) und des Augenhintergrundes (Affektion des N.opticus, erhöhter Liquordruck, Lipofuscin-flecken: EMND-Symptomatik) zur Untersuchung des Nervensystems am stehenden Tier.

\section{Beurteilung der Motorik auf hartem und tiefem Boden, bergauf- und bergabwärts an der Hand und unter dem Reiter}

Beurteilt wird Schritt und Trab, an der Hand, geradeaus und auf dem Zirkel auf hartem und weichem Boden, wenn möglich bergauf und bergab (Paresen: funktionelle Lahmheiten, Zehenschleifen, Schweiflähmung; Asynergien und Hypermetrien (Hahnentritt). Das Rückwärtsrichten an der Hand oder unter dem Reiter darf nicht vergessen werden (Asynergien, Hyperkinesien: Shivering). Unter Ataxie verstehen wir die Folge von Asynergie und Dysmetrie (zervikale-, zerebelläre-, vestibuläre - zerebrale A.). Die Belastung der Pferde im, dem Tier angepassten submaximalen Leistungsbereich während mindestens drei Minuten, deckt in den meisten Fällen pathologische Atemgeräuschen (Hemiparesis laryngis) auf. Bei der Interpretation der Untersuchungsergebnisse sind die physiologischen Variationen der neurologischen Symptomatik unbedingt mitzuberücksichtigen. Werden jedoch krankhafte Symptome vermutet, ohne dass sie zur Zeit der Untersuchnung einem Krankheitsbild zugeordnet werden können, muss man weitergehende Unter-suchungsmethoden berücksichtigen (Endoskopie (Luftsack), Blutanalysen (Vit.E; Borreliose; Borna u.a.) In jedem Falle muss der Verdacht im Untersuchungsbericht vermerkt und im Gespräch mit dem Auftraggeber ausgiebig erläutert werden. Eine schriftliche Garantie auf vermutete subklinisch evtl. auch auf saisonal oder progredient verlaufende Erkrankungen des Nervensystems (Kopftic; Hemiparesis laryngis) wird in den meisten Fällen von beiden Parteien als akzeptable Lösung anerkannt. Dies gilt im Besonderen auch für Erbkrankheiten des Nervensystems (zerebelläre Abiotrophie; Hemiparesis laryngis; Stereotypien) und solche bei denen das Nervensystem sekundär betroffen ist ( Kompressionssyndrom im Zervikal- und Rückenbereich u.a.).

Prof. Reto Straub

Klinik fürNutztiere und Pferde

Universität Bern

Bremgartenstraße 109

CH 3012 Bern 


\section{Untersuchung und Beur- teilung der oberen Atemwege - Die Endoskopie als Routinediagnostik?}

\author{
B. Ohnesorge \\ Klinik für Pferde, Tierärztliche Hochschule Hannover
}

\section{Einleitung}

Beim Tierkauf soll die sachverständige tierärztliche Untersuchung den Käufer vor Schaden bewahren und sicherstellen, dass das Pferd die im Kaufvertrag vereinbarten Eigenschaften, besonders Gesundheit und Leistungsfähigkeit besitzt. Dabei schuldet der Tierarzł dem Auftraggeber ein vollständiges und richtiges Gutachten über den Gesundheitszustand des Pferdes (Eikmeier et al. 1990). Durch die Änderung der gesetzlichen Gewährleistungfristen und den Wegfall der „Kaiserlichen Verordnung über Hauptmängel" erhält die tierärztliche Untersuchung und insbesondere auch die darauf basierende Befundbewertung eine weitreichende Bedeutung. Vor diesem Hintergrund soll die Notwendigkeit und Aussagefähigkeit einer endoskopischen Untersuchung im Rahmen einer Kaufuntersuchung betrachtet werden.

Grundsätzlich ist für die Protokollierung einer Kaufuntersuchung die Verwendung von vorgedruckten Durchschreibesätzen für die "Tierärztliche Untersuchung" empfehlenswert, wie sie von der Hippiatrika oder der Schlüterschen Verlagsgesellschaft im Handel sind. In diesen Vordrucken wird bereits in den "allgemeinen Bedingungen" festgestellt, dass der Umfang der Untersuchung vom Auftraggeber nach Beratung durch den Tierarzt zu bestimmen ist. Die endoskopische Untersuchung fällt dabei bisher unter die ergänzenden Untersuchungen, die über den im Protokoll vorgesehenen regulären Umfang hinausgehen und gesondert vereinbart werden müssen. Der Tierarzt sollte zu Beginn seiner Untersuchung auf die Möglichkeit einer endoskopischen Untersuchung der Atemwege und die damit verbundene Aussagefähigkeit hinweisen, auch, wenn er selbst nicht in der Lage ist, eine Endoskopie durchzuführen. Der Auftraggeber der Untersuchung kann dann entscheiden, ob er diese Untersuchung 1. grundsätzlich oder 2. unter bestimmten Voraussetzungen fordert, oder ob er sie 3. grundsätzlich ablehnt. Eine Endoskopie der Atemwege ist in jedem Fall dann dringend anzuraten, wenn sich im Rahmen der klinischen Untersuchung Hinweise auf eine Atemwegserkrankung ergeben. Wird in einem solchen Fall vom Auftraggeber keine Endoskopie erwünscht, so sollte über das Informationsdefizit aufgeklärt und die Entscheidung des Auftraggebers im Protokoll explizit vermerkt werden.

Zu bedenken ist, dass nicht alle Erkrankungen der Atemwege, die eine Bedeutung für den Wert oder die zukünftige Nutzung eines Pferdes besitzen, mit klinischen Symptomen einhergehen (Tabelle 1). Einige Erkrankungen lassen sich nur im Rahmen einer Endoskopie aufdecken, wie z.B. kleinere Zubildungen in der Nasenhöhle oder mitunter auch ein Epiglottis-Entrapment. Besonders problematisch sind angeborene Erkrankungen wie z.B. kleine Gaumenspalten oder Kehlkopfmissbildungen, die aufgrund von Nichtvorhandensein oder einer Fehlinterpretation von klinischen Befunden unerkannt bleiben, weil auf eine endoskopische Untersuchung verzichtet wird.

\section{Erkrankungen der oberen Atemwege}

Erkrankungen der oberen Atemwege können die Nasen-, Nasennebenhöhlen und das Siebbein, die Luftsäcke, den Pharynx und Larynx oder die Trachea betreffen. Klinische Symptome bei Erkrankungen in diesen Bereichen sind vergröBerte Mandibularlymphknoten, einseitiger Nasenausfluss (mukös, purulent, blutig, futtrig), Auftreibungen oder Verformung des Angesichtsschädels und ein abnormer Geruch der Ausatemluft. Von besonderer Bedeutung sind Atemgeräusche, die entweder bereits in Ruhe oder aber während der Belastungsuntersuchung auftreten, und die nahezu immer auf eine abnorme Einengung der oberen Luftwege deuten. Dem damit verbundenen, erhöhten Atemwegswiderstand muss eine mögliche Leistungsbeeinträchtigung beigemessen werden. Da diese Regionen im Inneren des Kopfes liegen und über die Nüstern zugänglich sind, stellt die endoskopische Untersuchung die wichtigste Methode in der ergänzenden Diagnostik dar.

Erkrankungen der Nasen-, Nasennebenhöhlen und des Siebbeins

In diesem Bereich dient die endoskopische Untersuchung insbesondere der Erkennung von Stenosen durch Missbildungen, Verformungen oder Zubildungen. So führen Tumore der Nasengänge (z.B. Fibrome, Myxome) bei entsprechender Größe zu einer erheblichen Einengung bis hin zum vollständigen einseitigen Verschluß. Eine besondere Art der Zubildung in den Nasen- und Nasennebenhöhlen des Pferdes ist das progressive Siebbeinhämatom, das seinen Ursprung im Siebbeinlabyrinth findet und sich durch spontan auftretende Epistaxis äußert. Eine häufige Erkrankung der Nasennebenhöhlen ist die Sinusitis, die sowohl primär als auch sekundär, dann als Folge einer Zahnerkrankung, auftreten kann. Die Amyloidose betrifft beim Pferd insbesondere die Schleimhäute des oberen Atemtraktes. Die Einlagerung von Amyloid führt zu einer Einengung der Nasengänge und zu einer starken Blutungsneigung bei Berührung der sichtbar veränderten Schleimhaut.

\section{Luftsäcke}

Luftsackerkrankungen sind klinisch gekennzeichnet durch eine Umfangsvermehrung im Bereich der Ganaschen, eine Schwellung der Lnn. mandibulares, überwiegend einseitigen Nasenausfluß und pharyngeale Stridores. Auch diese Erkran- 
kungen lassen sich endoskopisch diagnostizieren. Abszesse der medialen retropharyngealen Lymphknoten treten bei einer Infektion mit Streptokokken z.B. im Rahmen einer Druse auf. Die in der ventralen Luftsackwand liegenden Lnn. retropharyngeales med. führen zu charakteristischen Umfangsvermehrungen im Bereich des Lufsackbodens und damit zu einer Einengung des Pharynx von dorsal. Sowohl die spontane als auch die artifiziell herbeigeführte Entleerung von Luftsackabszessen führt zum Luftsackempyem. Dabei kommt es besonders bei Kopftiefhaltung zum eitrigen Nasenausfluß, den man endoskopisch bis zu den Luftsackklappen verfolgen kann. Unbehandelt kann es bei chronischen Luftsackempyemen zu Konkrementbildung kommen, die endoskopisch sichtbar im Luftsack liegen. Fohlen können an einer Luftsacktympanie erkranken, bei der es durch eine missgebildete Falte im Bereich der Luftsackklappe zu einer Ventilstenose mit Überblähung des betroffenen Luftsackes kommt. Luftsackmykosen äußern sich klinisch durch einseitiges Nasenbluten, das sturzartig zum Verbluten des Pferdes führen kann. Im Luftsack ist endoskopisch ein arrodierendes Pilzgranulom im Bereich der arteriellen Gefäße erkennbar.

\section{Pharynx}

Die Palatochisis ist eine angeborene Mißbildung des Gaumensegels, die durch milchigen bzw. futtrigen Nasenausfluß charakterisiert ist. Insbesondere kleiner Gaumenspalten im kaudalen Bereich des weichen Gaumens lassen sich nicht durch eine Betrachtung von der Maulhöhle sondern nur endoskopisch bei dorsal liegendem Gaumensegel erkennen. Die klinische Symptomatik kann bei derartig kleinen Gaumenspalten undeutlich sein, so dass die Erkrankung bei Verzicht auf eine Endoskopie verborgen bleibt. Bei der krankhaften dorsalen Verlagerung des Gaumensegels kommt es bei Belastung des Pferdes zu einem nahezu pathognomonischen, exspiratorisch schlotternden Atemgeräusch. Die Ursachen für diese habituelle, belastungsinduzierte Verlagerung sind häufig nicht sicher erkennbar, während die permanente Verlagerung durch Missbildungen des Gaumensegels oder der Epiglottis oder aber auch durch Innervationsstörungen bedingt wird.

Die follikuläre Hyperplasie der Pharynxschleimhaut wird häufig als Ursache für Atemgeräusche angesprochen. Sie tritt bei über 90\% der jungen Pferden auf und klingt in der Regel bis zum fünften Lebensjahr auch ohne Behandlung ab. Aufgrund der hohen Inzidenz kann das Vorhandensein einer follikuären Hyperplasie bei jungen Pferden nicht als krankhafter Befund gewertet werden. Dennoch besitzł diese Veränderung eine Bedeutung, wenn sie gemeinsam mit einer geringgradigen Kehlkopflähmung und einem hörbaren Atemgeräusch auftritt. In diesen Fällen kann häufig nicht sofort geklärt werden, ob das Atemgeräusch durch die follikuläre Hyperplasie oder durch die Kehlkopflähmung hervorgerufen wird. Solitäre Pharynxfollikel sind als einzelne verbliebene hyperplastische Follikel zu sehen.

Des weiteren kann eine Einengung des Pharynx durch eine Pharyngitis, durch eine Absenkung des Pharynxdaches im Rahmen von Luftsackerkrankungen und durch einen dynamischen Kollaps während der Belastung hervorgerufen werden.
Letztere können üblicherweise nicht während einer Endoskopie in Ruhe sondern erst bei einer endoskopischen Laufbanduntersuchung erkannt werden.

Larynx

Im Bereich des Larynx führen Entzündungen der Epiglottis zu einer Umfangsvermehrung des Kehldeckels und können mit Abszedierung oder Knorpelnekrose einhergehen. Neben einer angeborenen Epiglottishypoplasie kann auch eine derartige Entzündung zu einem verkleinerten Kehldeckel führen, so daß das Gaumensegel keinen Halt mehr findet und nach dorsal schlägt (s.o.). Beim Epiglottis-Entrapment wird der Kehldeckel durch eine Schleimhautfalte "eingefangen" und dadurch in der Bewegung beeinträchtigt. Schleimhautzysten treten im Pharynx meist als Subepiglottiszysten auf. Die Laryngitis kann akut und chronisch auftreten und ist meist ein Begleitsymptom einer Erkrankung der tiefen Atemwege. Ein Larynxödem wird im Rahmen des Morbus maculosus oder nach Traumen im Rachenraum (z.B. durch eine Nasenschlundsonde) beobachtet und führt nicht selten zu erheblicher Atemnot.

Die Hemiplegia laryngis ist in der überwiegenden Zahl der Fälle als Folge einer idiopathischen, erblich bedingten Neuropathie des N. laryngeus recurrens anzusehen (Ohnesorge et al. 1993). Nur in Einzelfällen tritt die Kehlkopflähmung sekundär als Folge einer traumatischen Nervenschädigung oder einer Tumorerkrankung auf. Die Bewertung der unterschiedlichen Grade einer Hemiplegia laryngis erhält im Unterschied zur bisherigen "Hauptmangelregelung" eine neue Bedeutung. So ist nunmehr auch zu berücksichtigen, in wie weit die Lähmung die Leistungsfähigkeit oder Zuchttauglichkeit beeinträchtigt oder voraussichtlich beeinträchtigen kann. Eine derartige prognostische Bewertung sollte, wie die Problematik bei der Bewertung von Röntgenbefunden zeigt, mit großer Zurückhaltung erfolgen. So fehlen bisher gesicherte Daten über die weitere Entwicklung der Erkrankung sowie über die Korrelation zwischen dem Grad der Lähmung und der Einschränkung der Leistungsfähigkeit. Aufgrund der Erblichkeit der Erkrankung kann jedoch der Zuchtwert des Pferdes als gemindert gelten (Ohnesorge et al. 1993). Der rostrale Vorfall des Arcus palatopharyngeus ist die endoskopisch sichtbare Folge einer angeborenen Missbildung des Schildknorpels und gilt als chronische und unheilbare Erkrankung des Kehlkopfes.

Über die Diagnose einer Erkrankung hinausgehend können bei einer Endoskopie im Rahmen einer Kaufuntersuchung auch evtl. erfolgte chirurgische Therapiemaßnahmen erkannt werden. So deutet z.B. ein unbeweglich abduzierter linker Aryknorpel auf eine durchgeführte Laryngoplastik sowie Narbenbildungen im Bereich der Stimmtaschen auf eine Ventrikelektomie hin.

\section{Trachea}

In der Trachea können Einengungen durch angeborene Missbildungen wie z.B. bei einer Säbelscheidentrachea oder auch durch erworbene Veränderungen wie z.B. nach Verletzungen und bei Zubildungen zu Atemgeräuschen führen. Die häufig 
vorhandenen sog. Spikula in der Trachea sind dagegen in ihrer Bedeutung zu vernachlässigen. In jedem Fall kann die Diagnose durch eine Endoskopie gesichert werden.

\section{Zusammenfassung}

Durch eine Endoskopie der oberen Atemwege können einerseits Erkrankungen erkannt werden, die sich nicht durch klinische Symptome äußern (Tabelle 1). Andererseits ermöglicht dieses Untersuchungsverfahren bei Vorliegen von klinischen Symptomen eine genavere Diagnostik. Bisher zählt die Endoskopie im Rahmen einer Kaufuntersuchung zu den ergänzenden, gesondert zu vereinbarenden Untersuchungsverfahren. Die Entscheidung für oder gegen die Durchführung einer Endoskopie sollte unter Berücksichtigung der klinischen Befunde, des Wertes des Pferdes und der Risikobereitschaft des Auftraggebers erfolgen. Dabei erscheint es sinnvoll, dass alle Vereinbarungen zu dieser Thematik nachvollziehbar im Untersuchungsprotokoll festgehalten werden. Wenig hilfreich wäre, die endoskopische Untersuchung grundsätzlich zum Bestandteil einer Kaufuntersuchung zu machen, da dadurch die Möglichkeit einer freien Vertragsgestaltung zwischen Tierarzł und Auftraggeber eingeschränkt würde. Die prognostische Bewertung von Endoskopie-Befunden sollte mit großer Zurückhaltung erfolgen, da die weitere Entwicklung von Befunden oder Krankheitssymptomen ähnlich wie bei der Bewertung von Röntgenbefunden in vielen Fällen nicht wissenschaftlich untersucht und unbekannt ist.

\section{Literatur}

H. Eikmeier, E. Fellmer und H. Moegle (1990): Lehrbuch der Gerichtlichen Tierheilkunde, Verlag Paul Parey, Berlin und Hamburg

B. Ohnesorge u.a. (1993): Hemiplegia laryngis bei Warmblutpferden - eine Untersuchung an Hengsten, Stuten und deren Nachkommen, J. Vet. Med. A 40, 134-154

Dr. Bernhard Ohnesorge

Klinik für Pferde

Tierärztliche Hochschule Hannover

Bischofsholer Damm 15

30173 Hannover

b.ohnesorge@tiho-hannover.de

\author{
B. Hertsch \\ Klinik für Pferde der Freien Universität Berlin
}

Erkrankungen aus dem Bereich des Halses und des Rückens sind beim Sportpferd sowohl als Folgen der Haltung, der Aufzucht, als auch der Nutzung in verschiedensten Formen und Graduierungen zu erwarten. Im Vordergrund der Schädigung stehen Weide- oder auch Sportunfälle mit einem akuten und auch die Nutzung des Pferdes deutlich beeinträchtigenden klinischen Bild. Ein derartiges offensichtlich erkennbares Leiden wird bei der Kaufuntersuchung sicherlich keine Rolle spielen. Hierfür kommen Zustände in Betracht, die entweder nur eine minimale klinische Folge haben, oder die als Reste einer ausgeheilten Grunderkrankung zu betrachten sind. Dazu gehören die Veränderungen insbesondere nach einer Spondylolisthesis mit den Folgen einer spinalen Ataxie, aber auch die Veränderungen der Spondylarthrose an den Halswirbeln mit oder ohne Ataxiefolgen, die Frakturen an den Gelenkflächen mit oder ohne Ataxiefolgen jedoch ebenso wie die Spondylarthrosen mit einer deutlich eingeschränkten Beweglichkeit der Halswirbelsäule. Diese Einschränkung ist als Problematik dann oft nur dem Reiter deutlich.

Wie kann und soll ein Tierarzt bei einer Kaufuntersuchung ein derartiges Problem aufdecken, sofern es sich um minimale 
klinisch relevante Befunde handelt?

Ähnlich verhält es sich mit den Erkrankungen aus dem Bereich des Rückens, sei es in Form von Frakturen, von Spondylarthrosen, von Kissing Spines, von Ankylosen unter mehr oder weniger starker Mitbeteiligung des Rückenmarkes, der Gelenke, der Bänder, der austretenden Nerven, der versorgenden Blutgefäße und der Muskulatur. Sofern sich die Problematik weniger in eindeutigen klinischen Symptomen als vielmehr reiterlichen Problemen äußert, wird der klinischmedizinische Nachweis einer Grunderkrankung sehr problematisch. Das heißt in solchen Fällen bestehen bereits deutliche reiterliche Probleme. Wie schwierig wird diese Problematik erst beim Kauf eines Pferdes, wo die Probleme von dem neuen Reiter noch nicht erkannt und von dem ehemaligen Reiter gut ausgeglichen sind?

Hierdurch droht eine Verlagerung des Problems Rittigkeit und Ausprobieren in das Gebiet der Kaufuntersuchung hinein. Nach meinem Dafürhalten ist das eine Problematik an die wir uns in keinem Fall heranwagen sollten. Diese Problematik ist nur zu lösen, wenn die Frage andersherum gestellt wird. Der Reiter hat ein Problem mit seinem Pferd und es gilt nun als Aufgabe den medizinischen Hintergrund dazu abzuklären. Nicht aber andersherum, dass bei der Kaufuntersuchung verdeckte Mängel als zukünftige reiterliche Probleme aufgedeck werden sollen. Hier endet eindeutig unsere Kompetenz, egal wie eng unsere Verbindung zum Pferdesport ist.

Die Untersuchungsmöglichkeiten sind, sofern man nur die sogenannte klinische Untersuchung ausschöpft, begrenzt. Zur klinischen Untersuchung im Bereich der Hals-, Brust- und Lendenwirbelsäule gehören neben der Adspektion und Palpation Manipulationen verschiedenster Art in begrenztem Umfang.

Die röntgenologische Untersuchung der Hals-, Brust- und Lendenwirbelsäule ist in mehr oder weniger großem Umfang möglich. Sie kann als wesentlicher Teil einer Erweiterung der Kaufuntersuchung auf Hals und Rücken betrachtet werden. Voraussetzung ist jedoch die Möglichkeit auswertbare Röntgenaufnahmen herzustellen. Sie müssen am stehenden Pferd anzufertigen sein. Röntgenaufnahmen oder andere Untersuchungen in Vollnarkose am liegenden Pferd halte ich auch bei Kaufuntersuchungen mit hohem finanziellem Hintergrund aus vielen Aspekten für unangebracht. Es ist damit zu rechnen, dass Röntgenaufnahmen der einzelnen Abschnitte der Wirbelsäule am stehenden Pferd nur mit Einschränkungen anzufertigen sind. So dass vorher eine Klarheit über Projektion, Darstellbarkeit und Auswertbarkeit getroffen sein muss.
Ein für mich denkbarer Standard wären seitliche Aufnahmen der gesamten Halswirbelsäule (1.-7. Halswirbel sowie die Dornfortsätze vom 5. bis zum 18. Brustwirbel und vom 1. bis zum 3. Lendenwirbel). Die Darstellung der kleinen Wirbelgelenke der Brust- und Lendenwirbelsäule am stehenden Pferd ist vorerst technisch für die Praxis ein nicht gelöstes Problem. Ebenso nicht ausführbar ist die $0^{\circ}$-Aufnahme der Halswirbelsäule. Die Graduierung der röntgenologisch feststellbaren Veränderungen an den Dornfortsätzen ist möglich und durchaus auch in das Schema der Röntgenbeurteilung im Sinne einer Kaufuntersuchung anzupassen.

Spezielle Untersuchungen, wie die Szintigraphie, Thermographie, Sonographie, Computertomographie, Kernspintomographie und weitere apparative Untersuchungen der Zukunft, sollten nicht Eingang in die Kaufuntersuchung finden, es sei denn es liegen gezielte und spezielle Aufträge zur Untersuchung vor. Die Übernahme eines derartigen Auftrages muss speziell insbesondere unter den Aspekten des Übernahmeverschuldens getroffen werden.

Labordiagnostische Untersuchungen im Rahmen von Kaufuntersuchungen zur Unterscheidung schmerzhafter Bänder oder Skeletterkrankungen von Myopathien können nur in Verbindung mit entsprechenden klinischen Befunden stehen, wenn sie sinnvoll gedeutet werden sollen. Für solche Fälle wird aber bereits der klinische Befund ausreichen. Denn wie bei allen Kaufuntersuchungen gilt nicht die Stellung einer Diagnose als Ziel, sondern die Erhebung von Befunden. Demzufolge sind auch diagnostische Lokalanästhesien bei Verdacht auf Rückenerkrankungen im Rahmen der Kaufuntersuchung nicht angezeigt.

Schlussendlich bleibt zu sagen, dass die klinische Untersuchung der Hals- und Rückenregion Eingang in die Kaufuntersuchung finden sollte, die röntgenologische Untersuchung kann ebenfalls einbezogen werden. Dazu müssen die Möglichkeiten und Grenzen der Röntgenuntersuchung klar aufgezeigt werden. Eine darüber hinausreichende Erweiterung des Untersuchungsumfanges sollte speziellen Aufträgen vorbehalten sein. Eine ständige Anpassung an den technischen Stand der Möglichkeiten der Untersuchung von Hals- und Rücken ist erforderlich.

Prof. Dr. Bodo Hertsch, Geschäftsführender Direktor

Pferdeklinik, Freie Universität Berlin

Oertzenweg 19b,

14163 Berlin

hertsch@vetmed.fu-berlin.de 


\section{Die Identifizierung und allgemeine Untersuchung des Pferdes}

\author{
A. Grabner \\ Klinik für Pferde, Allgemeine Chirurgie und Radiologie, Freie Universität Berlin
}

\section{Nationale (Kennzeichen, Signalement)}

Die Erfassung des Signalements ist zu Beginn jeder Untersuchung, so auch im Rahmen einer Kaufuntersuchung durchzuführen. Zur Kennzeichnung dienen die Farbe, das Geschlecht, das Alter, die angeborenen und erworbenen Abzeichen, die Körperhöhe (Stockmaß) und die Rasse mit Nutzungs- bzw. Verwendungszweck des Pferdes.

Zur Altersbestimmung werden Zahnbogen und Zahnwölbung, Durchbruch der Zähne, Verschwinden der Kunden, Kundenspur, Kernspur, Form der Kauflächen und die Galvaynesche Rinne herangezogen. Bei den angeborenen Abzeichen werden zuerst die im Bereich des Kopfes und in der weiteren Reihenfolge die Abzeichen an den Vorder- und HintergliedmaBen und dann im weiteren Bereich des Körpers erfasst. Erworbene Abzeichen sind Gestüts- und Nummernbrände, Tätowierungen, Druckflecke in Geschirr-, Halfter und Sattellage sowie Narben.

Bei Vorlage des Equidenpasses, in dem das Pferdediagramm durch eine individuelle Lebensnummer ergänzt wird, kann die vormals aufwändige und hinsichtlich der Abzeichen nicht immer präzise Vorgehensweise der Kennzeichnung als entbehrlich angesehen werden.

Neben diesen natürlich vorgegebenen, passiven Kennzeichen stehen bei verschiedenen Pferderassen, insbesondere Traber und Friesen auch aktive Kennzeichen in Form implantierter Transponder (Chips) zur Identifizierung zur Verfügung. Die Erkennung dieser Merkmale ist aber nicht prima vista, sondern nur über ein Lesegerät möglich.

\section{Allgemeine klinische Untersuchung}

Vor Beginn der Untersuchung sollte eine Anamnese des Auftraggebers vorliegen, die neben Eigentums- bzw. Besitzverhältnissen, Ausbildungs- und Trainingsstand des Pferdes, Angaben zu Abweichungen des Verhaltens mit Besonderheiten des Verhaltens im Stall oder bei der Nutzung, zur Haltung, Einstreu und Fütterung, zu Überempfindlichkeitsreaktionen (Haut, Respirationsorgane), zu früheren Erkrankungen, Operationen, Behandlungen und therapie-flankierenden Maßnahmen, zu Impfstatus und Entwurmungsregime und zur Vornahme des letzten Beschlags enthält.

Die allgemeine klinische Untersuchung (Status praesens) wird nach den Regeln der klinischen Propädeutik ausgeführt. Sie beinhaltet 12 zu prüfende klinische Parameter und ist dazu geeignet, erste Hinweise für eine Gesundheitsstörung und den Schweregrad der Beeinträchtigung des Allgemeinzustandes zu gewinnen.

\section{Parameter der allgemeinen Untersuchung}

Allgemeinverhalten

Körperhaltung und Gliedmaßenstellung

Allgemeinzustand und Habitus

Ernährungszustand

Pflegezustand

Rektale Körpertemperatur

Pulsfrequenz

Atemfrequenz

Adspektion der sichtbaren Schleimhäute

Prüfung der kapillären Wiederfüllungszeit (KFZ)

Palpation der Kehlgangslymphknoten (Lnn. mandibularis)

Auslösbarkeit des Hustens

Mit der Beurteilung des Allgemeinverhaltens gegebenenfalls unter Heranziehung anamnestischer Angaben zum Umgang unter verschiedenen individuellen und Umwelteinflüssen (Rasse, Alter, Geschlecht, Ausbildungsstand) findet eine erste Prüfung des Bewusstseins, der Sinneseindrücke und motorischer Reaktionen statt. Änderungen können sich in Verminderung (z. B. matt, teilnahmslos, schläfrig) oder Steigerung (z. B. übererregbar, schreckhaft) der Verhaltensäußerungen und in stereotypen Verhaltensweisen (z. B. Koppen, Weben) unterschiedlichen Grades kundtun. Physiologische Befunde sind für adulte Tiere "ruhig und aufmerksam" und für Fohlen und Jährlinge "lebhaft und aufmerksam". Zur richtigen Beurteilung sind gute Kenntnisse des physiologischen Verhaltenspektrums notwendig. Die Untersuchung erfolgt durch Adspektion, Nähertreten und Herbeiführen von Umwelteinwirkungen (Geräusche erzeugen, Aufzäumen, Vorführen des Pferdes).

Bei der Untersuchung der Körperhaltung werden Haltungsfehler, Veränderungen der Rückenlinie und der Gliedmaßenstellung sowie ein erhöhter oder verminderter Muskeltonus registriert. Entsprechende Hinweise auf Störungen können gegebenenfalls in einem neurologischen Untersuchungsgang genaver beurteilt werden.

Die Untersuchung des Ernährungszustandes erfolgt durch Adspektion und Palpation mit Prüfung der Augengruben, des Mähnenkamms, der Rippen, der Hüfthöcker und der Kruppe.

Bei der Untersuchung des Pflegezustands wird auf Glätte des Haarkleids, Staub, Schmutz und Parasiten zwischen den Haaren und auf Länge, Form und Beschaffenheit der Hufe und Zustand der Strahlfurchen geachtet.

Im Rahmen der Allgemeinuntersuchung werden Haarkleid und Haut durch Adspektion und Palpation mituntersucht.

Bei der Adspektion der Atmung werden neben Bestimmung der Atemfrequenz, Atemtypus (physiologisch kosto-abdominal), Rhythmus und Atemtiefe überprüft. Tiefe, erschwerte Atmung (Hyperpnoe und Dyspnoe) ist ein Hinweis auf eine Obstruktion der Atemwege; oberflächliche und schnelle Atmung (Hypopnoe und Tachypnoe) zeigen eine Restriktion der Lungenausdehnung durch Pneumonie oder Pleuraerguss an. Wichtige Symptome von Seiten des Respirationstraktes, 
wie Nasenausfluss und Husten können bereits bei der Allgemeinuntersuchung zur Feststellung kommen.

Die Untersuchung der Schleimhäute umfasst die Adspektion der Lidbindehaut, der Sklera, der Nasen- und Maulschleimhaut. Die physiologische Farbe der Konjunktiven ist rosa, der Nasen- und Maulschleimhaut blassrosa. Wichtige Informationen auf den Zustand des Kreislaufs, auf Hämokonzentration bzw. Anämie, auf den Bilirubinspiegel im Blut und auf Störungen der Blutgerinnung können durch diese Untersuchung gewonnen werden.

Die Untersuchung der Mandibularlymphknoten erfolgt durch Adspektion und Palpation beidseits im Kehlgang und Prüfung auf Größe, Konsistenz, Verschieblichkeit und Schmerzhaftigkeit. Abweichungen von der Norm weisen zumeist auf bakteriell-entzündliche Prozesse im Bereich des Nasopharynx und der Nebenhöhlen hin.

Im Anschluss an die Überprüfung der Kehlgangslymphknoten sollte eine beidseitige Palpation der oberen Halsgegend von ventral nach dorsal (Larynx-, Pharynx-, Parotis- und Luftsakkregion) mit Prüfung auf Schwellung, Umfangsvermehrung und Druckdolenz vorgenommen werden, da diese topographische Region ein hohes Befundpotential bietet.

\section{Klinische Organuntersuchung}

Hinsichtlich des Untersuchungsgangs gibt es prinzipiell eine Untersuchung nach Organsystemen und einen topographischen Untersuchungsgang, der alle Organe und Organsysteme in der jeweiligen Körperregion einbezieht. Für die Kaufuntersuchung von Vorteil ist ersterer mit einem systematischen Aufbau und der zusammenhängenden Darstellung eines Funktionskomplexes, z. B. Untersuchung des Kreislaufsystems: Darstellung der Konjunktiven und der episkleralen Gefäßinjektion

Pulspalpation an der A. facialis mit Prüfung von Frequenz, Qualität, Rhythmus, Gleichmäßigkeit, Gefäßfüllung und Gefäßspannung

Adspektion und Palpation der Jugularvenen beidseits mit Anstauprobe, Prüfung auf Verdichtungen, Blutangebot, Abfluss und pulsierende Wellen

Spezielle Herzuntersuchung mit Palpation der Herzregion und Auskultation zur Feststellung von Frequenz, Intensität und Abgesetztheit der Herztöne, Rhythmus und Herzgeräuschen

Obwohl die allgemeine Untersuchung des Pferdes im Rahmen einer Kaufuntersuchung nicht mit hoher Wahrscheinlichkeit klinische Befunde erwarten lässt, sollte diese verbindlich einer klinischen Organuntersuchung vorgeschaltet sein, da ihre Unterlassung, z. B. bei Fieber oder Schleimhautveränderungen forensische Konsequenzen nach sich ziehen kann.

\section{Literatur}

Baumgartner W (Hrsg.): Klinische Propädeutik der inneren Krankheiten und Hautkrankheiten der Haus- und Heimtiere, 4. Aufl. Parey Verlag, Berlin (1999)

F. E. I. und Deutsche Reiterliche Vereinigung (Hrsg.): Identifikation von Pferden. Schnell Buch \& Druck 4. Aufl., Warendorf (2000)

Speirs VC: Clinical examination of horses. WB Saunders, Philadelphia (1997)
Taylor FGR, Hillyer MH: Klinische Diagnostik in der Pferdepraxis. Schlütersche (2001).

Prof. Arthur Grabner

Klnik für Pferde, Freie Universität Berlin

Oertzenweg 19 b

14163 Berlin

grabner@vetmed.fu-berlin.de

\author{
A. Grabner \\ Klinik für Pferde, allgemeine Chirurgie und Radiologie \\ Freie Universität Berlin.
}

\section{Untersuchung des Kopfes}

Schon bei der äußeren Untersuchung des Kopfes können Hinweise auf pathologische Veränderungen in der Maulhöhle gewonnen werden. Bei der Adspektion wird auf Symmetrie, Umfangsvermehrungen, Verletzungen, Fistelöffnungen, Tränenflussspuren, auf die Kiefergelenkregion und den Ohrgrund geachtet. Die Palpation erstreckt sich auf die Kehlgangslymphknoten, die Unterkieferäste, die Lippen und die Rima oris. Stirnhöhle und Oberkieferhöhlen werden mit dem Finger perkutorisch untersucht. Auf das Vorliegen von Foetor ex ore (süßlich, faulig, kariös) wird geachtet.

\section{Untersuchung der Maulhöhle und des Gebisses}

Die Untersuchung des Digestionsapparates beginnt mit der Prüfung der Futter- und Wasseraufnahme und des Abschlukkens. Anamnestische Beobachtungen können dabei wertvoll sein. Es folgt eine Adspektion der labialen Anteile der Maulschleimhaut, des Frontzahnbogens (Dentes incisivi und Dentes canini) und der Zahnwölbung, der prämolaren und der molaren Zähne mit Prüfung auf angeborene Kiefer- und Zahnanomalien sowie erworbene Gebissveränderungen. Eine Zahnaltersbestimmung wird zur Bestätigung der Altersangabe im Equidenpass durchgeführt. Die grobvisuelle Untersuchung der Maulhöhle wird mit der Adspektion von Maulschleimhaut, Zahnfleisch, Zunge und Gaumen fortgesetzt, wobei besonders auf Schleimhautveränderungen und 
Umfangsvermehrungen geachtet wird.

Liegen Symptome, wie z. B. verlangsamte Futteraufnahme, Wickelkauen, Herausfallen von Hafer aus dem Maul, vermehrte Speichelbildung, Schleimhautläsionen, Blutungen, unvollständiger Kiefer- und Zahnreihenverschluss, Asymmetrie der Schädelknochen, Auftreibungen im Ober- und Unterkieferbereich vor, besteht eine Indikation für weitere Untersuchungen (z. B. feinvisuelle Adspektion der Maulhöhle unter Sedation, ggf. endoskopisch oder mit einer Interoralkamera, Verwendung von Zahnuntersuchungshaken, Röntgen) in Absprache mit dem Aufftraggeber.

\section{Untersuchung von Rachen, Schlund und Magen}

Bei der beidseitigen Palpation der Pharynxregion können bei Vorliegen von Druckdolenz und Kopfstrecken Hinweise auf schmerzhafte Veränderungen im Rachenbereich gewonnen werden, die durch die Endoskopie und gegebenenfalls durch Röntgen und Sonographie ergänzt werden müssen. Dies gilt auch für Schlund und Magen, da sich diese Organe weitgehend den rein klinischen Untersuchungsmöglichkeiten entziehen.

\section{Untersuchung des Abdomens}

Bei der Adspektion erhält man Hinweise auf das Vorliegen lokaler Umfangsvermehrung oder -verminderung, einer Ödembildung am Abdomen, im Präputial- oder Gesäugebereich oder einer Gasansammlung im Dickdarm. Durch die Palpation der Bauchdecke werden eine Spannungsänderung und Druckempfindlichkeit nachgewiesen und eventuelle Laparotomie-narben erkannt. Die Auskultation gibt durch Frequenz, Lautstärke und Klangcharakter informationen übe die aktuelle Peristaltik, die von der Konsistenz der Ingesta, der Menge der Darmgase und dem Durchmesser des Darmlumens abhängig ist. Besondere diagnostische Bedeutung hat bei der Auskultation des Abdomens, die regelmäßig über den 4 Quadranten vorgenommen wird, das ileozäkale Einspritzgeräusch im rechten oberen Quadranten.

Den Abschluss der äußeren klinischen Untersuchung des Abdomens bildet die Untersuchung der Afterregion und des
Kotabsatzes. Dabei wird auf Symmetrie des Anus, etwaige Umfangsvermehrungen, Kotverschmutzung (Diarrhoe) und Schleimfäden geachtet. Durch Palpation des Anus wird der Schließmuskel kontrolliert. Die Konsistenz, Farbe und Geruch des Kotes werden überprüft. Bei Vorliegen unphysiologischer Veränderungen sind weiterführende Untersuchungen (z.B.BU) angezeigt. Die Einleitung einer parasitologischen Kotuntersuchung wird im Rahmen der Kaufuntersuchung immer empfohlen.

\section{Rektale Untersuchung}

Bei der rektalen Untersuchung wird eine direkte Palpation des Rektums und eine indirekte Palpation von Organen und Strukturen im explorativen Bereich des Abdomens durchgeführt, deren Lage, Form und Konsistenz mehr oder weniger konstant bleibt (Leistenringe beim Hengst, Uterus und Ovarien bei der Stute, laterale Tänie der linken ventralen Kolonlängslage mit Beckenflexur, kaudaler Milzrand, Milz-NierenBand, kaudaler Nierenpol, kaudale Aufzweigung der Aorta abdominalis, vordere Gekrösewurzel, Caput caeci) oder variabel ist (Harnblase mit Füllungszustand, kaudales Gekröse mit Colon tenue, Corpus caeci mit Taenia ventralis caeci).

Die rektale Untersuchung ist ein Eingriff und sollte im Rahmen der Kaufuntersuchung nur nach entsprechender Indikation in Absprache mit dem Auftraggeber vorgenommen werden. Als solche sind klinische Befunde wie erhöhte Bauchdeckenspannung, abnorme Darmgeräusche und unphysiologische Kotkonsistenz zu nennen. Indikationen liegen auch bei anamnestisch geäußerter Disposition zu Koliken oder rezidivierender Diarrhoe vor. Eine rektale Untersuchung ist auch bei Kryptorchismus angezeigt und Gegenstand bei Untersuchungen auf Zuchttauglichkeit bei Stute und Hengst. Dagegen wird die rektale Untersuchung als Routinediagnostik im Rahmen der Kaufuntersuchung nicht empfohlen.

Prof. Arthur Grabner

Klnik für Pferde, Freie Universität Berlin

Oertzenweg 19 b

14163 Berlin

grabner@vetmed.fu-berlin.de 